\title{
Article
}

\section{HAWC+/SOFIA Multiwavelength Polarimetric Observations of OMC-1}

Chuss, David, Andersson, B.G., Bally, John, Dotson, Jessie L., Dowell, C. Darren, Guerra, Jordan A., Harper, Doyal A., Jones, Terry Jay, Lazarian, A., Ward-Thompson, Derek and Et, Al

Available at https://clok.uclan.ac.uk/27826/

Chuss, David, Andersson, B.G., Bally, John, Dotson, Jessie L., Dowell, C. Darren, Guerra, Jordan A., Harper, Doyal A., Jones, Terry Jay, Lazarian, A. et al (2019) HAWC+/SOFIA Multiwavelength Polarimetric Observations of OMC-1. The Astrophysical Journal, 872 (187). ISSN 0004-637X

It is advisable to refer to the publisher's version if you intend to cite from the work. http://dx.doi.org/10.3847/1538-4357/aafd37

For more information about UCLan's research in this area go to http://www.uclan.ac.uk/researchgroups/ and search for < name of research Group>.

For information about Research generally at UCLan please go to http://www.uclan.ac.uk/research/

All outputs in CLoK are protected by Intellectual Property Rights law, including Copyright law. Copyright, IPR and Moral Rights for the works on this site are retained by the individual authors and/or other copyright owners. Terms and conditions for use of this material are defined in the policies page.

\section{CLoK}

Central Lancashire online Knowledge www.clok.uclan.ac.uk

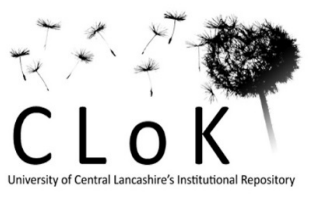




\title{
HAWC+/SOFIA Multiwavelength Polarimetric Observations of OMC-1
}

David T. Chuss $^{1}$ (1), B-G Andersson ${ }^{2,3}$ (1) John Bally $^{4}$ (1) Jessie L. Dotson $^{3}$ (1), C. Darren Dowell ${ }^{5}$, Jordan A. Guerra ${ }^{1}$ (1), Doyal A. Harper ${ }^{6,7}$, Martin Houde ${ }^{8}$ (10), Terry Jay Jones ${ }^{9}$ (1) , A. Lazarian ${ }^{10}$, Enrique Lopez Rodriguez ${ }^{2,3}$ (1) Joseph M. Michail ${ }^{1,11}$ (1), Mark R. Morris ${ }^{12}$ (10), Giles Novak ${ }^{13}$ (1) Javad Siah ${ }^{1}$, Johannes Staguhn ${ }^{14,15}$ (1), John E. Vaillancourt ${ }^{16}$ (1), C. G. Volpert ${ }^{17}$, Michael Werner ${ }^{5}$, Edward J. Wollack ${ }^{15}$ (잉, Dominic J. Benford ${ }^{18}$ (1) , Marc Berthoud $^{7}$, Erin G. Cox ${ }^{13}$ (1), Richard Crutcher ${ }^{19}$, Daniel A. Dale ${ }^{20}$ (1) , L. M. Fissel ${ }^{21}$ (10), Paul F. Goldsmith ${ }^{5}$ (1), Ryan T. Hamilton ${ }^{22}$ (1), Shaul Hanany ${ }^{23}$ (1) , Thomas K. Henning $^{24}$, Leslie W. Looney ${ }^{19}$ (D) , S. Harvey Moseley ${ }^{15}$, Fabio P. Santos ${ }^{24}$ (iD), Ian Stephens ${ }^{25}$ (D), Konstantinos Tassis ${ }^{26,27}$ (i), Christopher Q. Trinh ${ }^{28}$, Eric Van Camp ${ }^{13}$, and Derek Ward-Thompson ${ }^{29}$ (1) $($ HAWC + Science Team $)$

\footnotetext{
${ }^{1}$ Department of Physics, Villanova University, 800 E. Lancaster Ave., Villanova, PA 19085, USA; david.chuss@ villanova.edu 2 SOFIA Science Center/Universities Space Research Association, USA ${ }^{3}$ NASA Ames Research Center, M.S. N232-12, Moffett Field, CA 94035, USA Rd., Evanston, IL 60208, USA

\begin{abstract}
We report new polarimetric and photometric maps of the massive star-forming region OMC-1 using the HAWC + instrument on the Stratospheric Observatory for Infrared Astronomy. We present continuum polarimetric and photometric measurements of this region at 53,89, 154, and $214 \mu \mathrm{m}$ at angular resolutions of $5^{\prime \prime}, 8^{\prime \prime}, 14^{\prime \prime}$, and $19^{\prime \prime}$ for the four bands, respectively. The photometric maps enable the computation of improved spectral energy distributions for the region. We find that at the longer wavelengths, the inferred magnetic field configuration matches the "hourglass" configuration seen in previous studies, indicating magnetically regulated star formation. The field morphology differs at the shorter wavelengths. The magnetic field inferred at these wavelengths traces the bipolar structure of the explosive Becklin-Neugebauer/Kleinman-Low outflow emerging from OMC-1 behind the Orion Nebula. Using statistical methods to estimate the field strength in the region, we find that the explosion dominates the magnetic field near the center of the feature. Farther out, the magnetic field is close to energetic equilibrium with the ejecta and may be providing confinement to the explosion. The correlation between polarization fraction and the local polarization angle dispersion indicates that the depolarization as a function of unpolarized intensity is a result of intrinsic field geometry as opposed to decreases in grain alignment efficiency in denser regions.
\end{abstract}

Key words: ISM: clouds - ISM: magnetic fields - stars: formation 


\section{Introduction}

Located at a distance of $390 \mathrm{pc}$ (Kounkel et al. 2017), the Orion Nebula has been well studied as the nearest example of a region of massive star formation. The OMC-1 part of this complex is located behind an $\mathrm{H}$ II region that is ionized by the Trapezium cluster of O-B stars. The main feature on the west side of OMC-1 is the molecular ridge, which is oriented roughly north-south and contains the Becklin-Neugebauer (BN) object (Becklin \& Neugebauer 1967), a massive young stellar object, and the Kleinman-Low Nebula (KL) (Kleinmann \& Low 1967), which consists of molecular gas and dust surrounding additional massive stars.

The BN/KL region contains a bidirectional outflow (Allen \& Burton 1993) oriented approximately perpendicular to the molecular ridge and having a total kinetic energy of $2-6 \times 10^{47}$ erg (Bally et al. 2011). This outflow is traced by $\mathrm{CO}$ and $\mathrm{H}_{2}$ emission (Bally et al. 2011, 2017) and is thought to have been produced by the dynamical decay of stellar orbits near the center of the explosion roughly $500 \mathrm{yr}$ ago. This explosion has been identified with the same dynamical event that ejected several massive stars, including BN, from the core.

To the southeast of the molecular ridge and H II region created by the Trapezium stars is the Orion bar, which bounds the $\mathrm{H}$ II region and contains a photon dominated region at the boundary between the $\mathrm{H}$ II region and the molecular material. The dynamical importance of the magnetic field in OMC-1 is of interest, in part because of the relatively high ( $\sim$ milligauss) fields estimated in the region by previous studies (Johnston et al. 1989; Heiles et al. 1993; Pattle et al. 2017).

A key technique for studying magnetic fields in star-forming regions is far-infrared and submillimeter polarimetry (Hildebrand et al. 2000). Interstellar dust grains can become aligned with their long axis perpendicular to the magnetic field direction via a process known as radiative alignment torque (RAT; Dolginov \& Mytrophanov 1976; Draine \& Weingartner 1997; Lazarian \& Hoang 2007). In this scenario, an anisotropic radiation field at wavelengths less than the grain diameter imparts an angular momentum to the grains. For grains with paramagnetic bulk properties, solid-body rotation is traded for quantum spin-flips in the nuclei of the constituent atoms - lowering the total energy of the system while conserving angular momentum-a process known as the Barnett effect. The resulting magnetization of the grain causes the angular momentum of the grain to undergo Larmor precession around the external magnetic field direction and, under the continued radiative torques, to align the grain angular momentum with the field. Because grains preferentially rotate about their axis of greatest moment of inertia, the observed polarization direction is perpendicular to the magnetic field direction projected on the plane of the sky. In regions of extremely strong radiation fields (or for nonparamagnetic grains), the reference direction of the alignment can shift from the magnetic field ( $B$-RAT) to that of the radiation field $k$-vector $(k$-RAT) as discussed by Lazarian \& Hoang (2007).

Schleuning (1998) mapped OMC-1 using far-infrared polarimetry at $100 \mu \mathrm{m}$ and submillimeter polarimetry at $350 \mu \mathrm{m}$ with angular resolutions of $35^{\prime \prime}$ and $18^{\prime \prime}$, respectively. These authors suggested that the magnetic field in this region is highly ordered with a general direction oriented northwestsoutheast. The field also exhibits a "pinch" in the orthogonal direction. This "hourglass" shape has been interpreted to indicate that the star formation in OMC-1 is magnetically regulated. That is, the field supports the cloud against gravitational collapse in the direction perpendicular to the magnetic field direction. Vallée \& Bastien (1999) measured the polarization at eight positions in OMC-1 at $760 \mu \mathrm{m}$, finding a similar inferred magnetic field direction. Houde et al. (2004) presented a larger map of the OMC-1 region at $350 \mu \mathrm{m}$ and found general verification of the hourglass pattern. These authors also note that the polarization of the bar does not follow the hourglass shape and note the low polarization, suggesting poor grain alignment as an explanation.

More recently, Ward-Thompson et al. (2017) have measured the polarization at 850 microns with an angular resolution of $14^{\prime \prime}$ using the SCUBA-2 instrument on the James Clerk Maxwell Telescope (JCMT). These authors suggest that the low polarization in the bar could be due to variation in the magnetic field structure (e.g., a helical structure in the photon dominated region). They also measure a field parallel to the northwest filament and connect this result to the work of Soler et al. (2013), who identify a statistical trend of magnetic field direction perpendicular to dense filamentary structures and parallel to low-density filamentary structures as an indicator of sub-Alfvenic dynamics. Pattle et al. (2017) estimate the field strength from the $850 \mu \mathrm{m}$ data through the use of the DavisChandrasekhar-Fermi (DCF; Davis 1951; Chandrasekhar \& Fermi 1953) technique in combination with a technique related to unsharp masking to separate the turbulent contribution to the angular dispersion from the large-scale field. They find the resulting field strength to be $6.6 \pm 4.7 \mathrm{mG}$. These authors also conclude that the $\mathrm{BN} / \mathrm{KL}$ outflow is regulated by the field and that the outflow is not responsible for creating the hourglass geometry.

Tang et al. (2010) have measured polarization over a small area in the core of the $\mathrm{BN} / \mathrm{KL}$ region using the Submillimeter Array (SMA) with the highest resolution to date $\left(1^{\prime \prime}\right.$ at $870 \mu \mathrm{m})$. These results indicate that the grains are most likely magnetically aligned and that magnetic field structure has features below the typical resolution of single-dish polarimeters. Poidevin et al. (2011) mapped the region using visual and near-infrared (NIR) polarimetry of stars that mostly samples the magnetic field geometry in the foreground of OMC-1 at visual wavelengths and the lines of sight to bright, embedded sources such as BN at NIR wavelengths.

In this work, we present far-infrared polarimetry and photometry in four bands from 53 to $214 \mu \mathrm{m}$ from the Highresolution Airborne Wideband Camera-Plus (HAWC+; Harper et al. 2018) on the Stratospheric Observatory for Infrared Astronomy (SOFIA). Section 2 describes the data and signalto-noise cuts. Section 3 describes new maps of OMC-1 temperature, column density, and dust emissivity index based on spectral energy distributions (SEDs) created from the HAWC + photometry and complementary data sets. We also describe data cuts based on estimates of the effects of reference beam intensity for all four bands. We explore the polarization fraction as a function of intensity for all four HAWC + bands. Finally, we use the DCF technique (Davis 1951; Chandrasekhar \& Fermi 1953) to estimate field strengths in the $\mathrm{BN} / \mathrm{KL}$ region (Becklin \& Neugebauer 1967; Kleinmann \& Low 1967), the Orion bar, and the intercloud medium surrounding the Trapezium cluster. We examine the field geometry around the BN/KL explosion (Bally et al. 2011, 2017) as illuminated by the $53 \mu \mathrm{m}$ HAWC + data. We summarize our findings in Section 4. 


\section{Observations}

Photometry (total intensity) and polarimetry data on the OMC-1 region were obtained using the HAWC + camera on SOFIA (Harper et al. 2018). For the purpose of mapping total intensity, raster scans of the source in all four bands were done in 2016 December on SOFIA flight 354. The observing time per band ranged from 9 minutes at $53 \mu \mathrm{m}$ to 2 minutes at $214 \mu \mathrm{m}$. For each scan, the band-specific half-wave plate was in place for optical similarity of the photometric measurements to the polarization observations discussed later in this section. The scan photometry data were reduced using CRUSH V2.4.2ALPHA1 (Kovács 2006, 2008) with nondefault reduction options. In particular, the "bright" keyword was used to stop possible clipping of data near brighter regions, notably close to BN/KL. Different combinations of the "sourcesize" and "rounds" keywords were used to recover spatial scales beyond the default reduction. For the 53, 89, and $214 \mu \mathrm{m}$ bands, a "sourcesize" of 100 " was used. This keyword was not used for the $154 \mu \mathrm{m}$ band. The map-making process was iterated 20 times for $53 \mu \mathrm{m}, 70$ times for $89 \mu \mathrm{m}, 15$ times for $154 \mu \mathrm{m}$, and 100 times for $214 \mu \mathrm{m}$ by setting the "rounds" parameter. In addition, the "stability" parameter was only changed for the $214 \mu \mathrm{m}$ band to $3 \mathrm{~s}$ from the CRUSH default of $5 \mathrm{~s}$ to remove any large-scale emission remaining in the map. The final scan reductions have effective resolutions of $5.1,7 ! 9,14$ !" 0 , and 18 " 7 for the 53, 89, 154, and $214 \mu \mathrm{m}$ bands, respectively. Due to the relatively small fractional bandwidth of the filters, $\Delta \lambda / \lambda \approx 0.2$, no color corrections are made to the data. Based on the variance of HAWC + planet measurements (from scan mode, analyzed with CRUSH), we adopt a $15 \%$ calibration uncertainty for the 53,89 , and $154 \mu \mathrm{m}$ bands and $20 \%$ for the $214 \mu \mathrm{m}$ band.

Polarimetry data in the 53,154, and $214 \mu \mathrm{m}$ bands were obtained in 2017 October-November, and polarimetry at $89 \mu \mathrm{m}$ was performed in both 2016 December and 2017 OctoberNovember. Additional polarimetry data at $53 \mu \mathrm{m}$ were obtained in 2018 September. (See Table 1 for a summary of the band specifications.) Polarimetry observations were done using the standard chop-nod-dither observing method (Harper et al. 2018). The chop throw ranged between 7!.6 and 8!.0, and the chop/nod angle was $125^{\circ}$, measured west of north. The observing times were approximately $3.5,2.4,0.5$, and $0.5 \mathrm{hr}$ at $53,89,154$, and $214 \mu \mathrm{m}$, respectively. The data were reduced using the V1.3.0-BETA3 (2018 April) version of the HAWC + data reduction pipeline, with some particular settings and enhancements as noted below. Our $89 \mu \mathrm{m}$ maps are qualitatively very similar to the ones in the SOFIA Data Cycle System 2018 June data release but have some differences in detail in both the signal and noise maps. As is standard, HAWC+ obtained total intensity data in chop-nod mode simultaneously with the polarimetry data. We utilize these data for our study of polarization as a function of intensity in Section 3.5 to take advantage of the accurate registration of the polarization and intensity. For photometry data elsewhere in the paper, we use the maps from the scan mode, reduced with CRUSH as described above.

Due to an intermittent vacuum leak in the HAWC + instrument in 2016, the $89 \mu \mathrm{m}$ data on SOFIA flight 355 in 2016 December suffered from condensation of a helium film on the detectors; two fields to the east of $\mathrm{BN} / \mathrm{KL}$ and one to the west $(\sim 30 \%$ of all $89 \mu \mathrm{m}$ data) were affected. The presence of this helium increased the thermal time constants of the detectors, thereby changing the amplitude and phase response of the system to the $10 \mathrm{~Hz}$ chop. To calibrate these data, we measured the time constants of each detector from the $3 \mathrm{~Hz}$ internal calibrator flashes interspersed with the chop-nod-dither data and generated new phase and gain correction tables by scaling to $10 \mathrm{~Hz}$, assuming the detector time constant acts as a single-pole filter. As a result of this correction, we noticed a significant improvement in the internal consistency of the $89 \mu \mathrm{m}$ measurements, especially in Stokes $I$, for which the flight 355 data no longer produced noticeable artifacts in maps of $\chi^{2}$.

Our $\chi^{2}$-based analysis of observations of other, fainter targets indicated that the dither map products from the V1.3.0BETA3 pipeline have calculated noise uncertainty that is typically $\sim 25 \%$ below the true uncertainty; therefore, we have increased the uncertainties in the $I, Q$, and $U$ maps by this amount to compensate. This increase in the uncertainties has been found to be a satisfactory way of treating residual systematic effects (including correlated noise) in similar polarimetric systems (Novak 2011). Instrumental polarization based on "polarization skydips," with the median over the focal plane ranging from $1.8 \%$ to $2.0 \%$ across the bands (Harper et al. 2018), has been removed from the measurements for each pixel by subtracting the reduced Stokes parameters of the instrumental polarization from the measured parameters (Hildebrand et al. 2000). In the merging of the measurements into combined maps, we use relative background subtraction (three offsets applied to each input $I, Q$, and $U$ map to minimize the standard deviation of the output map) and smoothing with a Gaussian kernel having FWHM equal to half that of the diffraction-limited beam for each $\mathrm{HAWC}+$ band (Harper et al. 2018); both of these are standard parts of the pipeline.

To minimize isolated "spikes" present in the $I, Q$, and $U$ maps, we used a deglitching algorithm that operates in the map domain. Each measurement is compared with 20 neighboring measurements, for which the mean, spatial slope, and standard deviation are calculated. Measurements that differ by more than $3 \sigma$ (statistical) from the neighbor model are eliminated. Approximately $1 \%-3 \%$ of measurements were removed by the deglitcher.

We examined the telescope tracking data for each integration. Three integrations on SOFIA flights 450 and 454 with unstable, oscillatory tracking were discarded. Due to an error in the telescope control software, the two nod positions on flights 442,444 , and 447 were displaced by approximately $\pm 3^{\prime \prime}$ (northwest and southeast) from the desired position; this affects primarily the northern half of the $53 \mu \mathrm{m}$ map. The effective point-spread function is larger and asymmetric in that part of the map.

A signal-to-noise threshold of $p / \sigma_{p}>3$ was applied to the polarization maps and magnetic field analysis. This corresponds to a statistical uncertainty in position angle of $<10^{\circ}$. In all maps, polarization fractions have been debiased according to $p_{\text {debias }}=\sqrt{p^{2}-\sigma_{p}^{2}}$ (Serkowski 1974). Table 1 shows a summary of the spectral and spatial resolution along with the number of Nyquist sampled detections above $3 \sigma$ for the four polarimetry data sets. The polarization maps are shown in Figure 1. The vectors shown sample the maps with a spacing approximately equal to the beam size and have been rotated by $90^{\circ}$ relative to the electric field orientation to represent the inferred magnetic field angle as projected onto the plane of the sky. 
Table 1

Polarimetric Data Summary

\begin{tabular}{|c|c|c|c|c|c|}
\hline $\begin{array}{l}\text { HAWC }+ \text { Band } \\
\left({ }^{\prime \prime}\right)\end{array}$ & $\begin{array}{l}\text { Band Center } \\
\qquad(\mu \mathrm{m})\end{array}$ & $\begin{array}{l}\text { FWHM Bandwidth } \\
\qquad(\mu \mathrm{m})\end{array}$ & $\begin{array}{l}\text { Fractional Bandwidth } \\
\qquad(\cdots)\end{array}$ & $\begin{array}{l}\text { FWHM Beam Size } \\
\qquad\left({ }^{\prime \prime}\right)\end{array}$ & $\begin{array}{l}\text { Number of Vectors } \\
>3 \sigma, \text { Nyquist Sampled }\end{array}$ \\
\hline A & 53 & 8.7 & 0.16 & 4.9 & 15,808 \\
\hline $\mathrm{C}$ & 89 & 17 & 0.19 & 7.8 & 8939 \\
\hline $\mathrm{D}$ & 154 & 34 & 0.22 & 13.6 & 2387 \\
\hline $\mathrm{E}$ & 214 & 44 & 0.21 & 18.2 & 1880 \\
\hline
\end{tabular}

\section{Analysis}

\subsection{SEDs}

Obtaining a complete picture of OMC-1 requires understanding the environments in which dust grains reside. We have used new photometric measurements from $\mathrm{HAWC}+$ along with archival multiwavelength photometry data to produce improved SEDs for the OMC-1 region. The archival data used to constrain thermal emission include those from the Photodetector Array Camera and Spectrometer (PACS) (Poglitsch et al. 2010) and the Spectral and Photometric Imaging Receiver (SPIRE) (Griffin et al. 2010) instruments on the Herschel Space Observatory ${ }^{30}$ (Pilbratt et al. 2010) and the Submillimetre Common-User Bolometer Array2 (SCUBA-2) (Chapin et al. 2013; Dempsey et al. 2013; Holland et al. 2013) instrument operating on the JCMT. Up to $30 \%$ of the in-band radiation in the SCUBA-2 $850 \mu \mathrm{m}$ data consists of free-free emission caused by UV from the Trapezium cluster (S. Coudé 2019, private communication). Therefore, we use also data from the MUltiplexed Squid TES Array at Ninety GHz (MUSTANG) (Dicker et al. 2008) on the Green Bank Telescope (GBT) and combined Very Large Array (VLA) and GBT $X$-band data to constrain the effects of freefree emission at longer wavelengths.

\subsubsection{Herschel Data}

Herschel data were obtained from the Herschel Science Archive $\left(\mathrm{HSA}^{31}\right)$ for the 70,100 , and $160 \mu \mathrm{m}$ PACS photometry bands and the $250 \mu \mathrm{m}$ SPIRE band. PACS observed the OMC-1 region with its $70 \mu \mathrm{m}$ filter on 2010 February 23 (OBSID 1342191106, 1342191107) with a 20" $\mathrm{s}^{-1}$ scan speed and high-gain photometer setting. The PACS 100 and $160 \mu \mathrm{m}$ filters observed OMC-1 on 2010 October 8 (OBSID 1342206052, 1342206053) with the same gain and scan speed settings used for the $70 \mu \mathrm{m}$ data. We used the level 2.5 maps made with the scanamorphos routine (Roussel 2013). A SPIRE level $2.5250 \mu \mathrm{m}$ map was used for this region, which merged observations from OBSID 1342184386 and 1342239930 and utilized a cross-scan mode with a scanning velocity of $30^{\prime \prime} \mathrm{s}^{-1}$. We do not utilize the SPIRE 350 and $500 \mu \mathrm{m}$ maps for OMC-1 due to their large beam size $\left(25^{\prime \prime}\right.$ and $36^{\prime \prime}$, respectively).

The angular resolution of each PACS band is dependent upon the scan speed of the individual observations and can be fitted as a two-dimensional Gaussian; the major and minor axes of this Gaussian are listed in Poglitsch et al. (2010). We use the geometric mean of these axes as the resolution of the band. For PACS, we adopt resolutions of 5!"6, 6." 8 , and 11 !" 3 for the 70 ,

\footnotetext{
30 Herschel is an ESA space observatory with science instruments provided by European-led Principal Investigator consortia and with important participation from NASA.

31 http://archives.esac.esa.int/hsa/whsa/
}

100 , and $160 \mu \mathrm{m}$ bands, respectively. We follow Sadavoy et al. (2013) in adopting a SPIRE $250 \mu \mathrm{m}$ resolution of 18 ". 2 .

Due to the wide passbands of the PACS and SPIRE photometer filters and the assumption of a flat SED (Müller et al. 2011; Valtchanov 2017), small color corrections $(\lesssim 7 \%)$ are made to the data. Müller et al. (2011) and the HIPE program (v15.0.1 with SPIRE calibration v.14_3 Ott 2010) list pretabulated color corrections for various modified SEDs. One point to note is that the values listed in Müller et al. (2011) are multiplicative-inverse corrections, while those in HIPE are multiplicative only. To use the pretabulated color corrections, we assume $\beta=2$. This is close to that determined by Vaillancourt (2002), who finds a median temperature and dust emissivity index, $\beta$, in this region of $40 \pm 10 \mathrm{~K}$ and $1.8 \pm 0.05$, respectively. For each PACS filter, we take the inverse of the color corrections listed for $\beta=2$ and $T=30-50 \mathrm{~K}$ to make them multiplicative factors and then adopt the rms of these values as the factor; we take the error on this correction to be the rms error of the values. For the 70, 100, and $160 \mu \mathrm{m}$ bands on PACS, these color corrections are 1.025, 1.004, and 0.929 , with errors of $0.004,0.018$, and 0.027 , respectively. For the SPIRE $250 \mu \mathrm{m}$ band, the process is the same without taking the inverse of the listed factors. Using the extended source corrections, we adopt a value of 0.970 with an error of 0.005 for the color correction. Finally, we follow Arab et al. (2012) and Sadavoy et al. (2013) in adopting calibration uncertainties of $20 \%$ for the PACS data and $10 \%$ for the SPIRE data, respectively.

\subsubsection{SCUBA-2 Data}

The SCUBA-2 instrument is able to simultaneously observe in two filters (Holland et al. 2013). For this work, we choose to only use the $850 \mu \mathrm{m}$ data, as the $450 \mu \mathrm{m}$ band has large calibration uncertainties $(\sim 50 \%)$ due to the high variabilty of the atmosphere at this wavelength (Sadavoy et al. 2013) compared to $850 \mu \mathrm{m}$.

In addition to possible free-free contamination, Coudé et al. (2016) and Mairs et al. (2016) find molecular contamination in the $850 \mu \mathrm{m}$ data from the ${ }^{12} \mathrm{CO}(J=3 \rightarrow 2)$ rotational line of up to $20 \%$. Coudé et al. (2016) note that areas of lower column density will likely have a higher contamination level of this line. To correct for this, we utilized archival CO-corrected data ${ }^{32}$ (Mairs et al. 2016) that made use of data from the HARP instrument (Buckle et al. 2009). We adopt a 14!"2 resolution for these corrected data (Mairs et al. 2016). While Sadavoy et al. 2013 adopt a $10 \%$ calibration uncertainty for the SCUBA-2 $850 \mu \mathrm{m}$ data, we adopt a $15 \%$ error to account for any additional systematic effects in the HARP instrument (Buckle et al. 2009).

\subsubsection{MUSTANG and X-band Data}

The MUSTANG (90 GHz; $3.3 \mathrm{~mm}$ ) and $X$-band $(8.4 \mathrm{GHz}$; $3.5 \mathrm{~cm}$ ) data used here were originally published in Dicker et al. (2009), which describes the reduction process. For the

\footnotetext{
32 http://www.cadc-ccda.hia-iha.nrc-cnrc.gc.ca/
} 

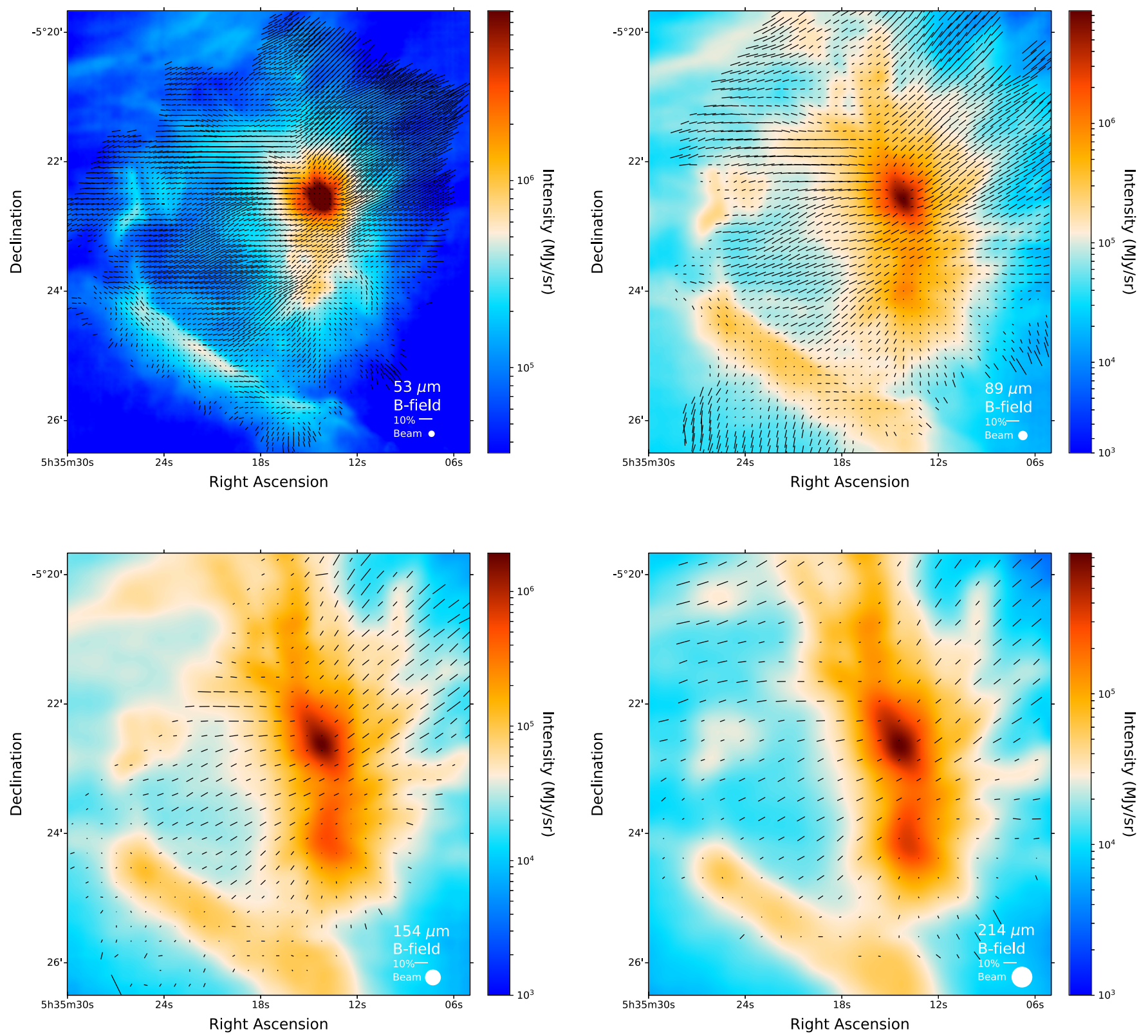

Figure 1. Polarization maps at 53,89,214, and $154 \mu \mathrm{m}$, clockwise starting from the top left. The polarization vectors have been rotated by $90^{\circ}$ to indicate the inferred direction of the magnetic field projected in the plane of the sky. Polarization data are from chop-nod HAWC + observations. The background intensity images are obtained using scan data and reduced with the CRUSH analysis software. The vectors are plotted such that their spacing is equal to the beam size.

MUSTANG data, we adopt a beam size of $9^{\prime \prime}$, and for the $X$ band data, we use 8." 4 for the beam size. For both instruments, we follow Dicker et al. (2009) in adopting a calibration uncertainty of $15 \%$.

\subsubsection{Data Preparation}

Arab et al. (2012) correct for zero-point emission in the PACS data by subtracting the intensity in a region around $\alpha=5^{\mathrm{h}} 35^{\mathrm{m}} 26^{\mathrm{s}} 7, \delta=-5^{\circ} 26^{\prime} 4^{\prime \prime} 7$ (J2000). We also correct for a zero-point; however, we set our zero value to that of the pixel located at this position. We propagate the error in quadrature to take this offset into account. The SPIRE data were zerocorrected in the default SPIRE HSA pipeline using Planck HFI/IRAS data as described in Bernard et al. (2010).

We do not apply zero-point corrections to the HAWC + and SCUBA-2 data due to their respective reduction methods.
CRUSH removes residual DC offsets and systematic, correlated sky noise (Kovács 2008) within a scan, and thus any arbitrary zero-point is already removed from the data. The SCUBA-2 reduction method (Starlink SMURF software) similarly removes a zero-point (Sadavoy et al. 2013).

The photometry and errors are color corrected, if applicable, and then are converted into common units of $\mathrm{MJy} \mathrm{sr}^{-1}$. Poglitsch et al. (2010) provide extended-source saturation levels for each PACS filter when using high-gain observations; any remaining pixels above these limits in the PACS images that were not flagged by the default PACS HSA pipeline were subsequently removed. To be more conservative on possible saturation, we enlarge the PACS $70 \mu \mathrm{m}$ map mask, which is then used to mask the PACS 100 and $160 \mu \mathrm{m}$ data near the $\mathrm{BN} / \mathrm{KL}$. The masked regions near $\mathrm{BN} / \mathrm{KL}$ cover $6.7,6.0$, and $6.0 \operatorname{arcmin}^{2}$ for the 70,100 , and $160 \mu \mathrm{m}$ maps, respectively. 
Table 2

Adopted Photometry Calibration Values

\begin{tabular}{|c|c|c|c|c|c|c|}
\hline $\begin{array}{l}\text { Observatory/ } \\
\text { Instrument } \\
\qquad(\cdots)\end{array}$ & $\begin{array}{l}\text { Wavelength } \\
\qquad(\mu \mathrm{m})\end{array}$ & $\begin{array}{c}\text { Beam Size } \\
\text { FWHM } \\
\left({ }^{\prime \prime}\right)\end{array}$ & $\begin{array}{c}\text { Color } \\
\text { Correction } \\
(\cdots)\end{array}$ & $\begin{array}{l}\text { Color Correction } \\
\text { Uncertainty } \\
(\cdots)\end{array}$ & $\begin{array}{c}\text { Calibration } \\
\text { Uncertainty } \\
(\%)\end{array}$ & $\begin{array}{l}\text { Paper Reference } \\
\qquad(\cdots)\end{array}$ \\
\hline SOFIA/HAWC + & 53 & 5.1 & $\ldots$ & 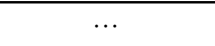 & 15 & This paper \\
\hline SOFIA/HAWC + & 89 & 7.9 & $\ldots$ & $\ldots$ & 15 & This paper \\
\hline SOFIA/HAWC + & 154 & 14.0 & $\cdots$ & $\cdots$ & 15 & This paper \\
\hline SOFIA/HAWC + & 214 & 18.7 & $\ldots$ & $\ldots$ & 20 & This paper \\
\hline Herschel/PACS & 70 & 5.6 & 1.025 & 0.004 & 20 & Abergel (2010) \\
\hline Herschel/PACS & 160 & 11.3 & 0.929 & 0.027 & 20 & André (2007) \\
\hline Herschel/SPIRE & 250 & 18.2 & 0.970 & 0.005 & 10 & André (2011), Bendo et al. (2013) \\
\hline JCMT/SCUBA-2 & 850 & 14.2 & $\cdots$ & $\cdots$ & 15 & Mairs et al. (2016) \\
\hline GBT/MUSTANG & 3500 & 9.0 & $\ldots$ & $\ldots$ & 15 & Dicker et al. (2009) \\
\hline GBT and VLA & 35000 & 8.4 & $\cdots$ & $\cdots$ & 15 & Dicker et al. (2009) \\
\hline
\end{tabular}

The photometry and error arrays were independently reprojected into a common WCS system with 3!.7 square pixels using a flux-conserving algorithm. Finally, an errorweighted Gaussian convolution was performed, with kernel size $\sqrt{\mathrm{FWHM}_{2}^{2}-\mathrm{FWHM}_{1}^{2}}$, where $\mathrm{FWHM}_{1}$ is the resolution of the each instrument and $\mathrm{FWHM}_{2}$ is the target common resolution of 18 ".7. A summary of the data used to generate the SEDs is given in Table 2.

\subsubsection{Temperature, $\beta$, and Column Density Maps}

The data are fit in two steps. First, we use the MUSTANG and $X$-band data to fit the free-free emission at longer wavelengths assuming the form given in Hensley et al. (2015)

$$
I_{\mathrm{ff}}=C\left(\frac{\nu}{30 \mathrm{GHz}}\right)^{-0.12},
$$

where $C$ is a normalization constant. The free-free emission contamination at $850 \mu \mathrm{m}$ is extrapolated from the fit and removed. Then, using a single-temperature modified blackbody curve (Equation (2)) as defined in the Appendix of Vaillancourt (2002), the thermal component,

$$
I_{\nu}=\left(1-e^{-\tau(\nu)}\right) B_{\nu}(T),
$$

is fit.

We define the optical depth, $\tau(\nu) \equiv \varepsilon\left(\nu / \nu_{0}\right)^{\beta}$, where $\varepsilon$ is a constant of proportionality directly related to the column density along the line of sight, $\beta$ is the dust emissivity index, and $B_{\nu}(T)$ is the Planck blackbody function at wavelength $\nu$ with temperature T. Following Sadavoy et al. (2013) (and references therein), we adopt $\nu_{0}=1000 \mathrm{GHz}$. From the Python Scipy package, we use curve fit to fit the function by minimizing the $\chi^{2}$ statistic using the Levenberg-Marquardt algorithm. We limit the data used in the fits to those points where the signal-to-noise is greater than 3 and fit all pixels for which the number of degrees of freedom is greater than 1 . The vast majority of fitted pixels $(\sim 87 \%)$ have a reduced $\chi^{2}\left(\chi_{r}^{2}\right)$ of 5 or less, with $\sim 70 \%$ having $\chi_{r}^{2} \leqslant 2$. Therefore, we apply a cut to the parameter maps to include only pixels for which $\chi_{r}^{2}<5$. We further apply a cut for $\beta<2.25$ as a proxy to eliminate edge pixels where data set limitations cause the fits to be suspect despite having reasonable $\chi_{r}^{2}$.
Comparing our definition of $\varepsilon$ with the modified blackbody function in Sadavoy et al. (2013), we find

$$
\varepsilon=\kappa_{\nu_{0}} \mu m_{\mathrm{H}} \mathrm{N}\left(\mathrm{H}_{2}\right) .
$$

Here, $\kappa_{\nu_{0}}$ is a reference dust opacity per unit mass at frequency $\nu_{0}, \mu$ is the mean molecular weight per hydrogen atom, $m_{\mathrm{H}}$ is the atomic mass of hydrogen, and $\mathrm{N}\left(\mathrm{H}_{2}\right)$ is the gas column density (molecules per square centimeter) (Sadavoy et al. 2013). We adopt the values $\kappa_{\nu_{0}}(1000 \mathrm{GHz})=0.1 \mathrm{~cm}^{2} \mathrm{~g}^{-1}$ and $\mu=2.8$ as in Sadavoy et al. (2013). Example SEDs and fits for three regions of OMC-1 are shown in Figure 2.

Maps of the fitted parameters are shown in Figure 3. The parameter maps have a median temperature and $\beta$ in this region of $36.3 \pm 2.5 \mathrm{~K}$ and $1.7 \pm 0.15$, respectively, which are consistent with the results in Vaillancourt (2002). The warmest region outside of the $\mathrm{BN} / \mathrm{KL}$ region $(83.9 \pm 19.3 \mathrm{~K})$ lies 1 1!9 southeast of the $\mathrm{BN}$ object or about $1^{\prime}$ southeast of the Trapezium cluster's center. Compared to Vaillancourt (2002), we find a temperature at this point that is $\sim 60 \%$ higher at our 18 ". 7 resolution, and $36 \%$ higher when smoothing the temperature map to a $30^{\prime \prime}$ resolution to match the angular resolution of this previous work. Similarly, at the location of $\mathrm{BN} / \mathrm{KL}$, this author find a temperature and $\beta$ of approximately $50 \mathrm{~K}$ and 1.5 , respectively. We find $T \approx 92.3 \pm 11.5 \mathrm{~K}$ and $\beta \approx 1.02 \pm 0.15$ at our 18 ". 7 resolution and $T \approx 80.4 \pm 1.0 \mathrm{~K}$ and $\beta \approx 1.20 \pm 0.02$ when smoothed to a $30^{\prime \prime}$ resolution. Thus, this disagreement in the fitted parameters cannot be described by a difference in resolution alone.

Across the mapped region, the value of $\beta$ is strongly and negatively correlated to the fitted temperature as shown in Figure 4. This is similar to the trend found by Dupac et al. (2001, 2003) for the OMC-1 region. Shetty et al. (2009) argue that this degeneracy could be due to line-of-sight variations. These authors also note that noise in the observations can cause such a degeneracy in the $T-\beta$ relation that is more apparent when using only data on the Rayleigh-Jeans tail of the SED than when the peak of the thermal spectrum is constrained.

To check whether the correlation in Figure 4 is an artifact of the fit (i.e., to explicitly search for systematic covariance between $T$ and $\beta$ ), we have refit the SEDs for the three fiducial regions shown in Figure 2 using a Markov Chain Monte Carlo (MCMC) technique (Foreman-Mackey et al. 2013; ForemanMackey 2016). Figure 5 shows the results from the MCMC for the three regions in Figure 2 and one additional region at the 

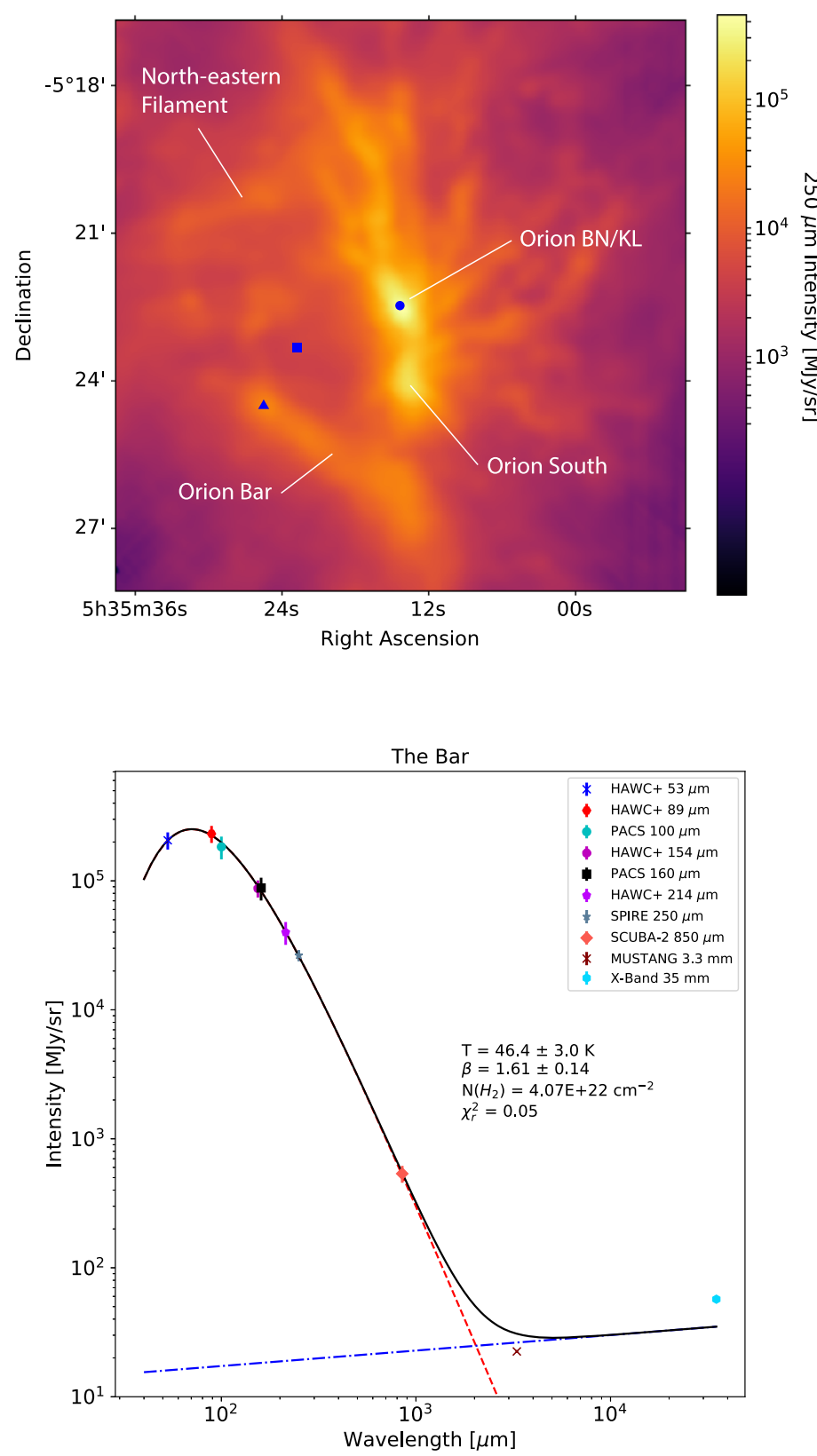
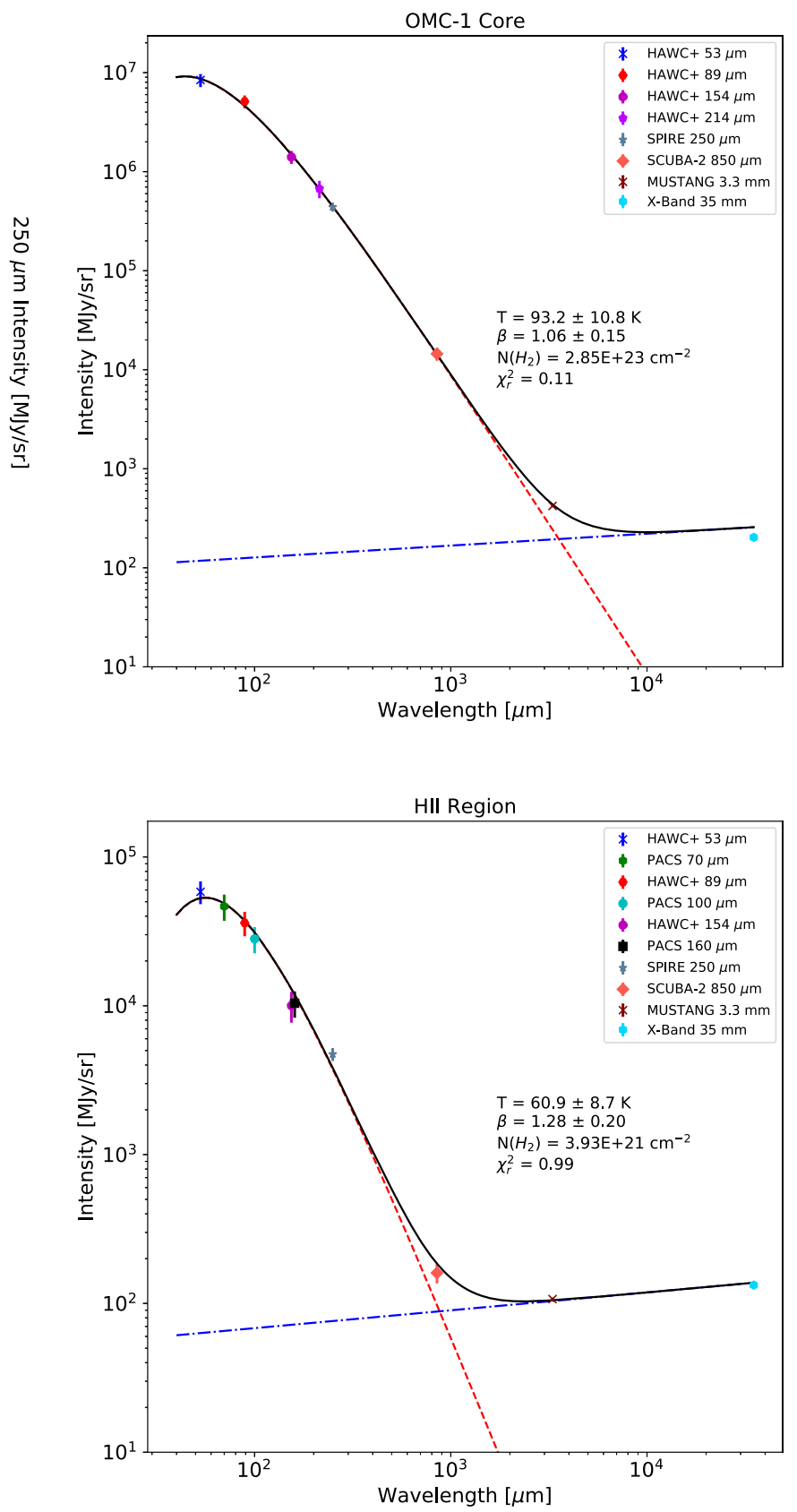

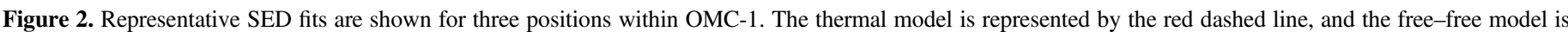

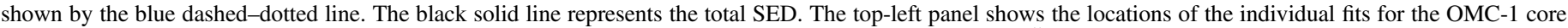
(circle), H II region (square), and bar (triangle) overplotted on the the SPIRE $250 \mu \mathrm{m}$ data smoothed to 18 ". 7 resolution.

edge of the map $\left(\alpha=5^{\mathrm{h}} 35^{\mathrm{m}} 5^{\mathrm{s}} \cdot 62, \delta=-5^{\circ} 21^{\prime} 14{ }^{\prime \prime} 35 ; \mathrm{J} 2000\right)$ where $T$ is low and $\beta$ is high. There is some covariance between $T$ and $\beta$; however, the width of the likelihood function agrees with the uncertainties obtained from the initial fits, giving confidence that the reported uncertainties remain reasonable despite the underlying covariance. Our likelihoods are similar to the distributions shown in Figure 3 of Galametz et al. (2012) in which the variation in fit parameters resulting from Monte Carlo modifications to the spectral data points is explored. We conclude from these arguments that the $T-\beta$ correlation observed in our mapped region likely has a physical origin, as opposed to being entirely an artifact of the fitting process.

\subsection{General Features}

To compare the inferred magnetic field directions between the maps at different wavelengths, we construct line integral contour (LIC) maps (Cabral \& Leedom 1993), as shown in Figure 6. The general direction of the magnetic field is similar in all four bands and is oriented perpendicular to the integral shaped filament (ISF). The hourglass pinching (Schleuning 1998; Houde et al. 2004; Ward-Thompson et al. 2017) of the magnetic field lines is apparent in all bands, albeit with less curvature than has been observed at $850 \mu \mathrm{m}$ (Pattle et al. 2017; Ward-Thompson et al. 2017). This feature has been identified as evidence of magnetically regulated collapse of the molecular cloud. 

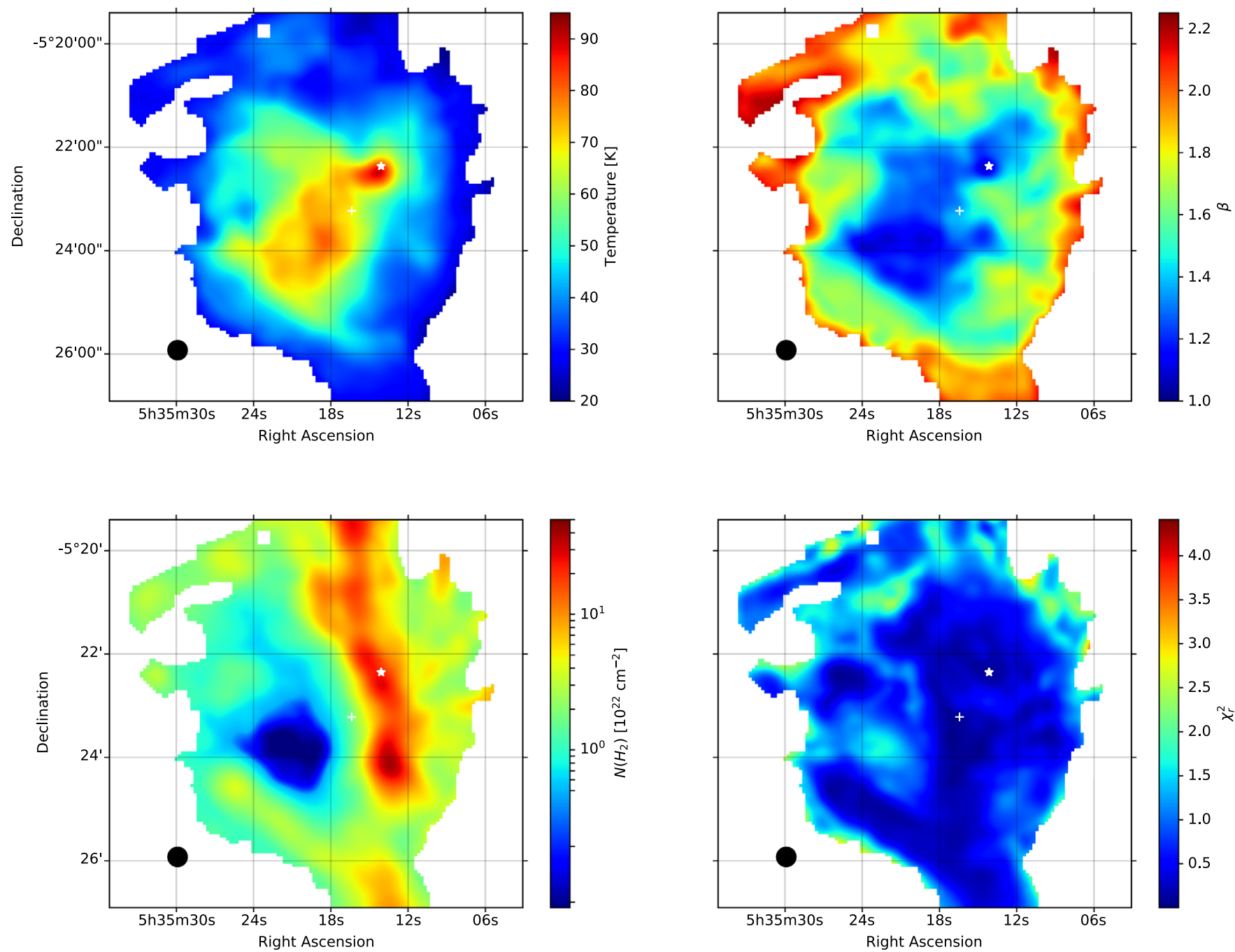

Figure 3. Clockwise from top left: the fitted temperatures, dust emissivity indices, $\chi_{r}^{2}$ values, and calculated column densities. All are smoothed to a $22^{\prime \prime}$ resolution. The effective beam size is shown on the bottom left in each panel as a black filled circle. Only points with $\chi_{r}^{2}<5$ are shown. The white cross indicates the center of the Trapezium cluster, while the white star shows the location of the BN object.

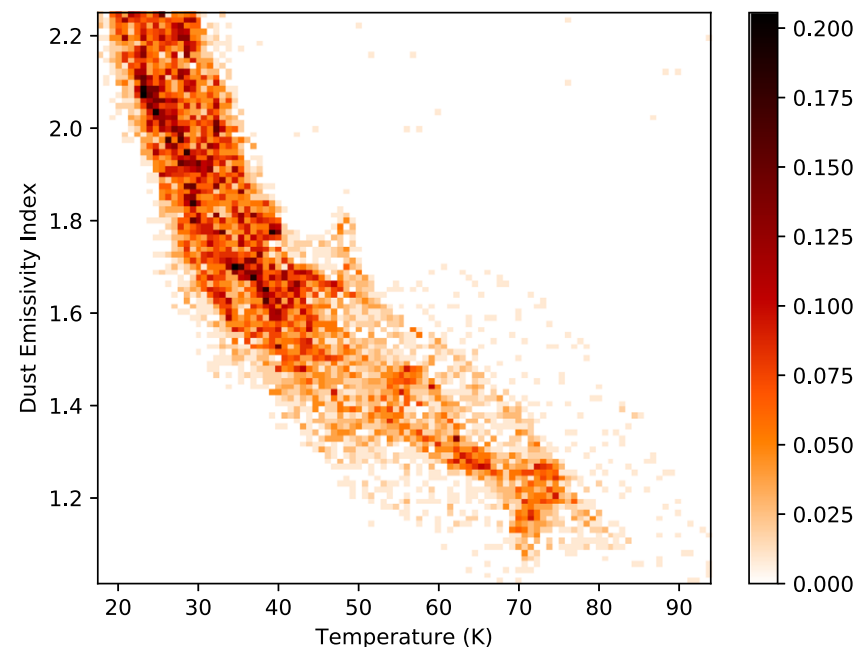

Figure 4. A two-dimensional histogram of dust emissivity index ( $\beta$ ) vs. temperature across the region shows an anticorrelation between the two quantities.

The inferred magnetic field direction near $\mathrm{BN} / \mathrm{KL}$ differs between the short and long wavelength bands. At 53 and $89 \mu \mathrm{m}$, there is a component of the field near the highest density region of the cloud that is parallel to the ISF. We suggest that this may be a result of the $\mathrm{BN} / \mathrm{KL}$ explosion compressing the fields perpendicular to the explosion direction. This feature is not seen at 154 or $214 \mu \mathrm{m}$. The inferred field through the center of $\mathrm{BN} / \mathrm{KL}$ in these bands is perpendicular to the ISF. This may be because the dust grains that dominate the emission at these wavelengths are cooler dust outside the region of the explosion. NIR (absorption) polarimetry of $\mathrm{BN}$ (Poidevin et al. 2011), which samples all of the dust along the line of sight to $\mathrm{BN}$ independent of dust temperature, yields a position angle of $115^{\circ}$ counterclockwise from north, in good agreement with our longer wavelength results. Note that the NIR fractional polarization of $\mathrm{BN}$ is normal for the amount of extinction (Jones 1989), indicating that the total column of dust in front of $\mathrm{BN}$ does not show the effects of strong depolarization.

The inferred magnetic field in the less dense material to the west of the ridge is generally consistent with the northwestsoutheast magnetic field structure (perpendicular to the ISF).

The polarization measured in the bar is considerably lower than in other regions in OMC-1, which is in agreement with longer wavelength measurements (Houde et al. 2004; WardThompson et al. 2017). This may indicate that the magnetic 

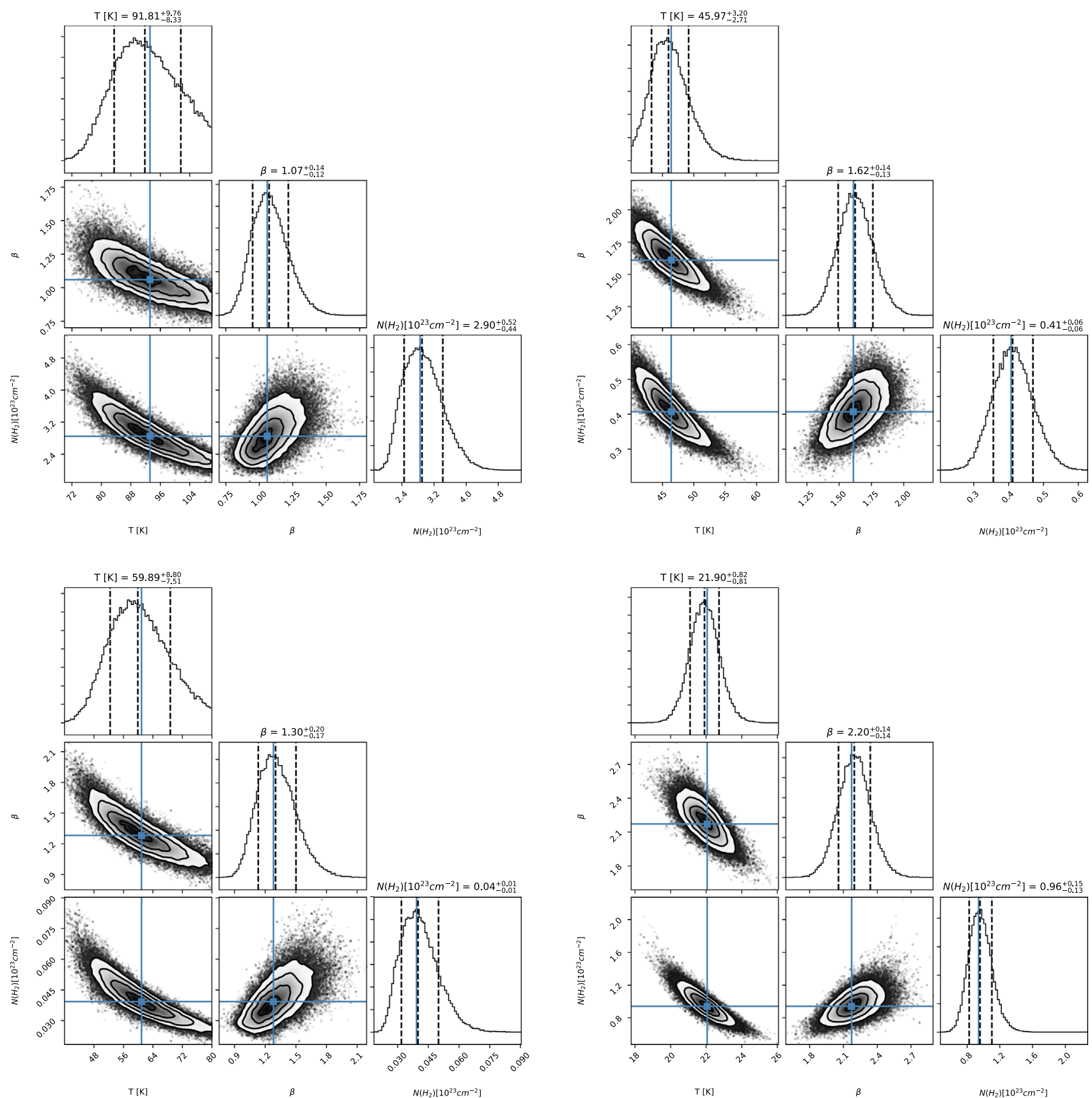

Figure 5. MCMC fits to four fiducial positions shown in Figure 2: core (top left), bar (top right), H II (bottom left), and an edge location of the map (bottom right, $\alpha=5^{\mathrm{h}} 35^{\mathrm{m}} 5^{\mathrm{s}} \cdot 62, \delta=-5^{\circ} 21^{\prime} 14^{\prime \prime}$. $35 ; \mathrm{J} 2000$ ). Results from the initial fit described in the text are superposed on the likelihood distributions and are found to be in agreement with the MCMC results. Dashed lines on either side of the maximum likelihood value (reported uncertainties in the plot titles) correspond to the $68 \%$ confidence limits $(1 \sigma)$.

field is mostly projected along the line of sight, that the grain alignment efficiency is low, that there is significant variation in the direction of the polarization within the beam, or some combination of these effects. Specifically, the low polarization fraction may be a result of polarization cancellation due to multiple magnetic fields superposed along the line of sight. For example, the ambient field of the cloud (running approximately northwest-southeast as observed in the H II region) could be superposed on a field that is parallel to the geometry of the bar, such as what one may expect from compression of the magnetic field if the bar is indeed an edge-on region bounding a bubble created by the Trapezium cluster (Dotson 1996; Novak et al. 2000).
This interpretation assumes that the grains are magnetically aligned. In regions where the grains are exposed to particularly high radiation fields, there is the possibility of radiative alignment ( $k$-RAT; Lazarian \& Hoang 2007). In this case, the angular momentum of the dust grains can become aligned with the $k$-vector direction of the radiation rather than with the magnetic field. Larger, cooler grains would be predominantly aligned by this process. The longer wavelength observations would manifest this effect preferentially compared to shorter wavelengths, and the effect would be strongest closest to the radiation source. The relationship between the Trapezium cluster and the Orion bar presents such a situation. In the $\mathrm{H}$ II region between the Trapezium cluster and the bar, the polarization direction (perpendicular to the 

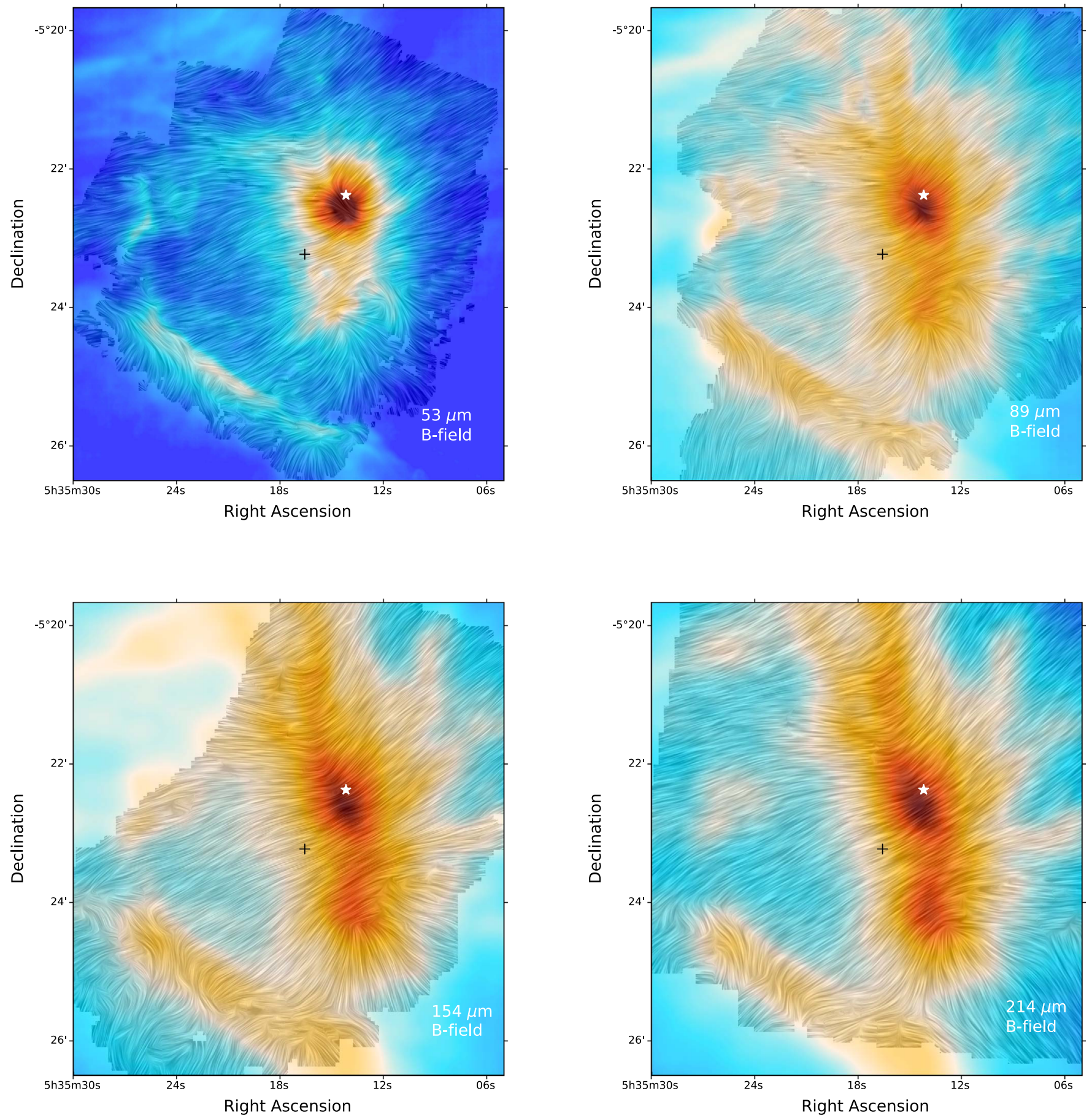

Figure 6. LIC (Cabral \& Leedom 1993) maps for the 53, 89, 214, and $154 \mu \mathrm{m}$ inferred magnetic field directions, clockwise starting from the top left. Polarization data are from chop-nod HAWC + observations. The background intensity images are the same as those used in Figure 1. In each image, the star indicates the location of $\mathrm{BN} / \mathrm{KL}$, and the cross indicates the location of the Trapezium cluster.

$B$-field lines in Figures 1 and 6) is such that the grains' angular momentum is roughly aligned with the $k$-vectors from the Trapezium cluster. This is the direction expected for radiative alignment. In the center of the bar (which is the region closest to the Trapezium cluster), the polarization direction changes between the $53 \mu \mathrm{m}$ data and the longer wavelengths. At $53 \mu \mathrm{m}$, the polarization is perpendicular to the $k$-vectors from the Trapezium cluster. At longer wavelengths, the polarization direction in the center of the bar is aligned with the polarization direction in the $\mathrm{H}$ II region. This observation is in tentative agreement with $k$-RAT theory.
Alternative explanations for this wavelength-dependent polarization direction include the possibility that polarized reference beam intensity could be altering the direction of the polarization around the bar. This effect is more significant at longer wavelengths (see Section 3.4). In addition, different wavelengths can be preferentially probing different regions along the line of sight, which may have different field geometries.

The polarized intensity $(p \times I)$ maps are shown in Figure 7 . In all bands, the polarized intensity is concentrated toward the center of field, just as is total intensity, suggesting that aligned 

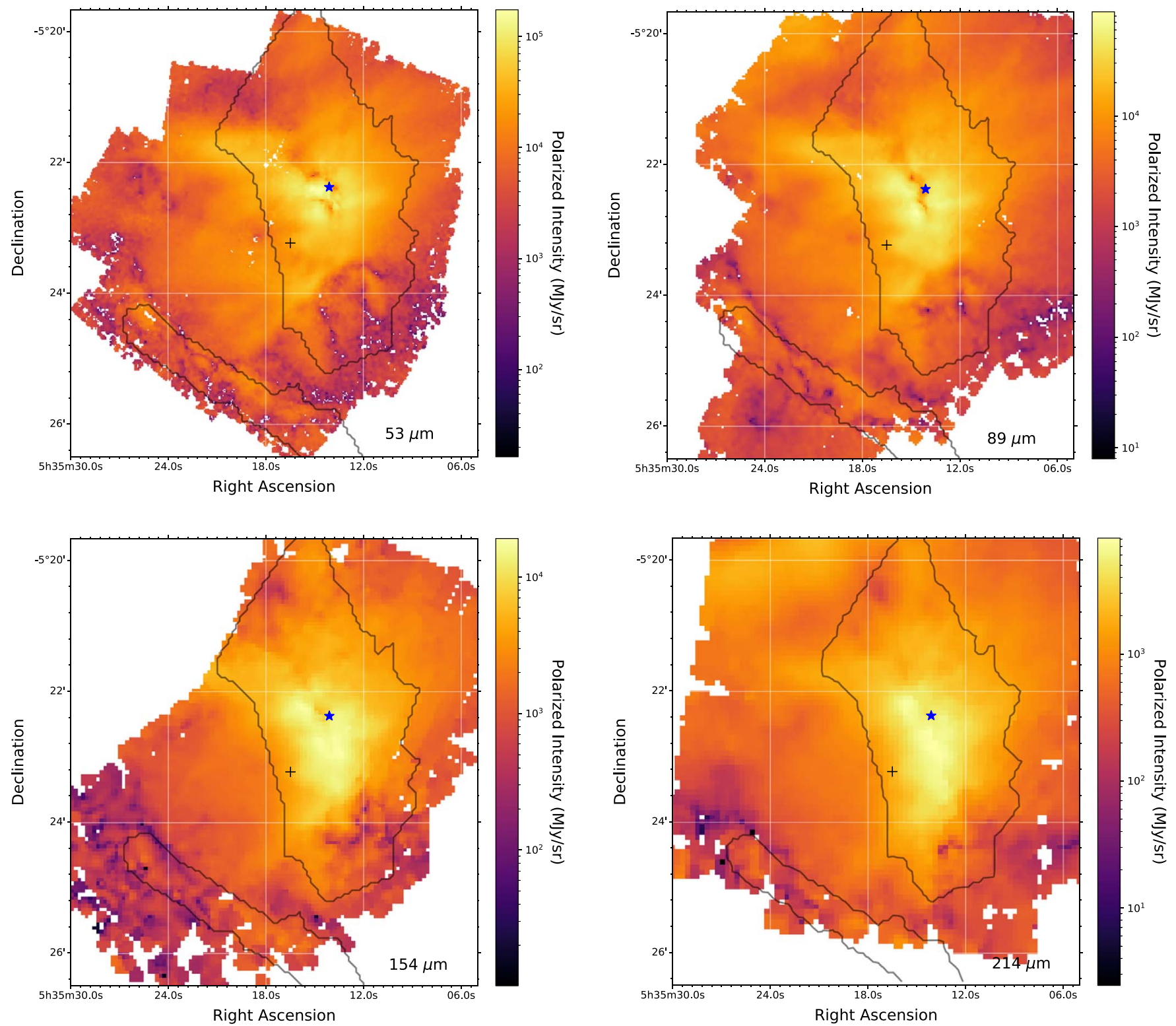

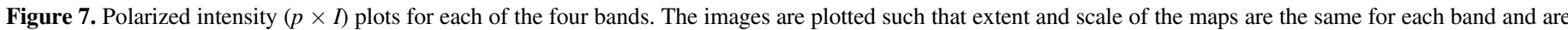

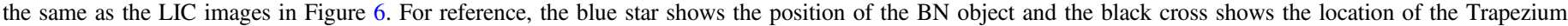
cluster. Masks defining the $\mathrm{BN} / \mathrm{KL}$ and bar regions are shown in light gray contours for reference (see Section 3.3).

dust grains are present throughout the region. Some features in polarized intensity correspond closely to features in the total intensity, but others do not. In the 53 and $89 \mu \mathrm{m}$ maps, there are regions of low polarized intensity coincident with the center of the $\mathrm{BN} / \mathrm{KL}$ explosion. They are not seen in the longer wavelengths, possibly because the larger beams do not resolve them. These low polarized intensity features can be explained by either magnetic field spatial variations at scales below that of the beam size, a geometry in which the magnetic field is predominantly oriented along the line of sight, or the effects of optical depth. The $89 \mu \mathrm{m}$ image shows this "depolarization" as a line oriented approximately perpendicular to the $\mathrm{BN} / \mathrm{KL}$ explosion axis (see Section 3.7). This may indicate that the depolarization is related to the explosion.

As is the case with the fractional polarization, the polarized intensity is also low near the bar. In this case, a thin line of low polarized intensity is observed in each of the four maps, which is located at the edge of the bar that is closest to the Trapezium cluster. This supports the idea that the field is aligned with the edge of the bar and is predominantly oriented along the line of sight, perpendicular to the ambient field along the line of sight, or a combination of the two.

\subsection{Object Masks}

In addition to the signal-to-noise threshold that is applied to the polarization maps, we apply additional cuts to our data for subsequent analysis. Temperature, density, and environmental conditions vary across the OMC- 1 complex. Thus, we anticipate that the magnetic field strength will as well. Because of this and the relatively large number of vectors in the HAWC + data, it is advantageous to apply analysis techniques to regions that are physically similar. Motivated by this, we have constructed a mask set that distinguishes three particular 


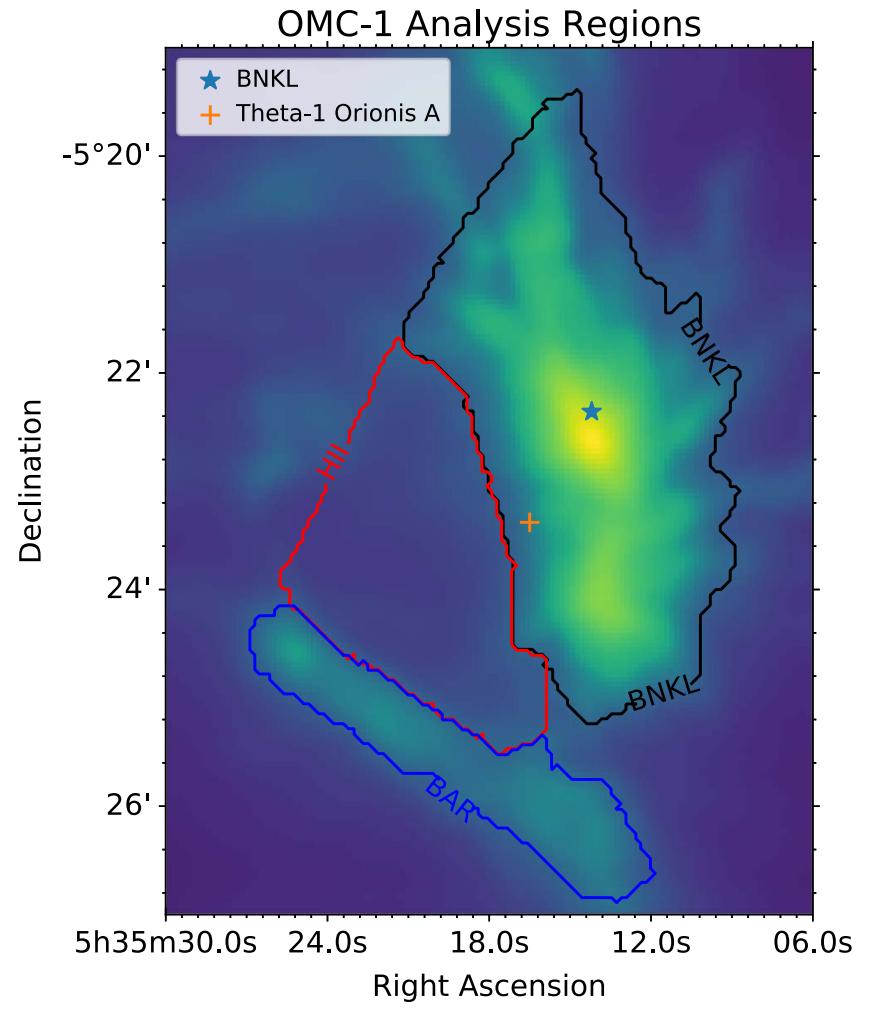

Figure 8. Three masked regions are indicated by the black (BNKL), red (H II), and blue (BAR) lines.

regions of interest. These regions correspond to 1 . the $\mathrm{BN} / \mathrm{KL}$ region and molecular ridge, 2 . the Orion bar, and 3. the less dense intercloud H II region that is heated by the Trapezium cluster of O-B stars. These regions will be denoted "BNKL," "BAR," and "H II," hereafter. The BNKL and BAR regions are defined by the $1700 \mathrm{MJy} \mathrm{sr}^{-1}$ contour in the HAWC $+154 \mu \mathrm{m}$ chop-nod photometry map. The H II region is defined as the area between the two other masks. These masks are shown superposed on the $154 \mu \mathrm{m} \mathrm{HAWC}+$ scan map in Figure 8.

\subsection{Reference Beam Contamination}

Because OMC-1 is located in a region of extended emission, it is necessary to quantify the effect of polarized emission in the reference beams of the observations. The measured normalized Stokes parameters, $\left(q_{m}, u_{m}\right)$, are related to the source polarization $\left(q_{s}, u_{s}\right)$, the reference polarization, $\left(q_{r}, u_{r}\right)$, the source intensity, $I_{s}$, and the mean intensity in the two reference beams, $\bar{I}_{r}$.

$$
\begin{aligned}
& q_{m}=\frac{q_{s} I_{s}-q_{r} \bar{I}_{r}}{I_{s}-\bar{I}_{r}}, \\
& u_{m}=\frac{u_{s} I_{s}-u_{r} \bar{I}_{r}}{I_{s}-\bar{I}_{r}} .
\end{aligned}
$$

Here we assume that the reference beam polarization fraction is the same in each reference beam because we will estimate a worst-case scenario by choosing a high but reasonable polarization fraction to make a pixel-by-pixel estimation of the maximum contamination. In this paper, we follow the formalism described in the Appendix of Novak et al. (1997) to estimate the maximum effect of reference beam contamination based on measured intensities and estimates of polarization of the reference beam contamination. The estimates for minimum and maximum limits to the fractional polarization $\left(p_{\mathrm{sys}}^{+}\right.$and $p_{\mathrm{sys}}^{-}$, respectively) and the maximum angular error can be written in terms of the ratio of the reference beam intensity to the measured intensity, $w \equiv \bar{I}_{r} / I_{m}$, the measured polarization, $p_{m}$, and the assumed reference beam polarization, $p_{r}$.

$$
\begin{gathered}
p_{\mathrm{sys}}^{+}=\max \left[p_{m},\left(\frac{p_{m}+p_{r} w}{1+w}\right)\right], \\
p_{\mathrm{sys}}^{-}=\frac{p_{m}-p_{r} w}{1+w}, \\
\Delta \phi_{\mathrm{sys}}=\frac{1}{2} \arctan \left[\frac{p_{r} w}{\left(p_{m}^{2}-p_{r}^{2} w^{2}\right)^{1 / 2}}\right] .
\end{gathered}
$$

We use a subset of the Herschel maps described in Section 3.1 to estimate $I_{r}$ for each pixel in the HAWC+ chop-nod maps. We use the Herschel maps to fit a simple model for the emission,

$$
I=A \nu^{2} B_{\nu}(T)
$$

where $A$ is a fitted amplitude and $B_{\nu}(T)$ is the Planck function. We then use this model to calculate the intensity for both the right and left reference beams for each observed sky pixel. The value of $\bar{I}_{r}$ is then found by averaging these two quantities. For the 53 and $89 \mu \mathrm{m}$ maps, we used the Herschel/PACS 100 and $160 \mu \mathrm{m}$ maps to model the intensity; for the 154 and $214 \mu \mathrm{m}$ maps, we added the Herschel/SPIRE $250 \mu \mathrm{m}$ maps when fitting for the intensity model. No smoothing was done for the Herschel maps.

The measured intensity in each band, $I_{m}$, was taken from the calibrated Stokes $I$ value of the HAWC + chop-nod maps. From these, a map of $w \equiv \bar{I}_{r} /\left(I_{s}-\bar{I}_{r}\right)$ was produced for each band. We assumed that the polarization of the reference beams is $p_{r}=0.10$ in all HAWC + bands. This represents one of the higher measurements of polarization observed and thus provides a conservative estimate. The values for the measured polarization, $p_{m}$, are the nondebiased polarization fractions from the HAWC + polarimetry data sets.

From these estimates of $w, p_{r}, p_{m}$, and $\bar{I}_{r}$, maps of the quantities in Equations (6)-(8) were made. In all bands, maps of these relevant quantities were stored in FITS format and then applied for subsequent data cuts. Figure 9 shows histograms of the upper and lower uncertainties on polarization due to estimated reference beam contamination along with corresponding histograms for the statistical errors $\left(\sigma_{p}\right)$ in each band.

As an illustration of this method, Figure 10 shows a mask set corresponding to the cut $\Delta \phi_{\text {sys }}<10^{\circ}$. This is the mask set utilized in Section 3.6. The $3 \sigma$ data cut is also included. The $53 \mu \mathrm{m}$ map is mostly unaffected by the cuts for possible reference beam contamination due to the higher temperatures of the OMC-1 center relative to the surrounding cloud. At longer wavelengths $(89,154$, and $214 \mu \mathrm{m})$, much of the bar region is flagged by this algorithm due to its low polarization. As noted in Section 3.2, there are multiple possible causes for this. 

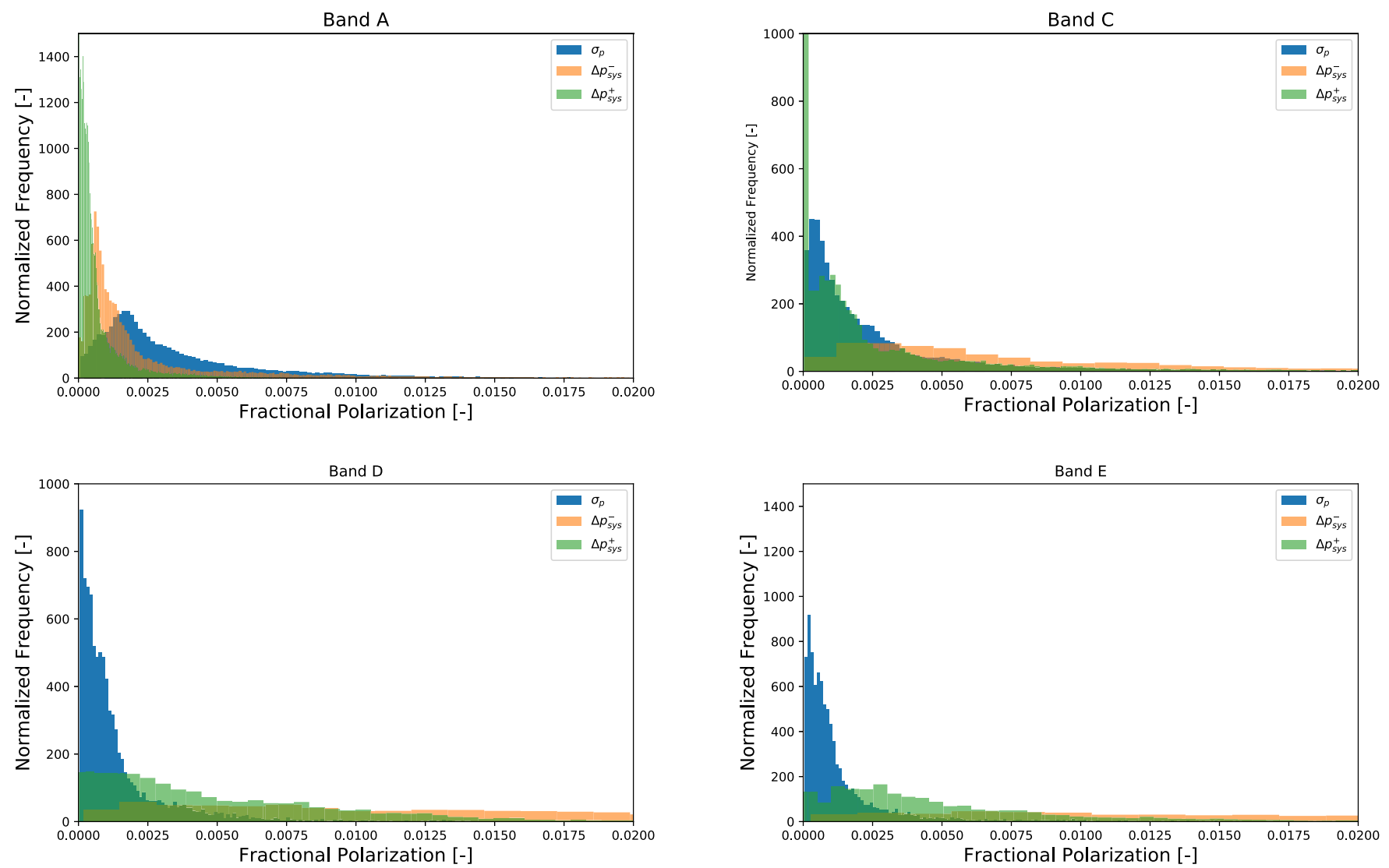

Figure 9. Histograms for the statistical errors on polarization fraction along with the upper and lower systematic errors for polarization fraction due to estimated reference beam contamination are shown for each of the four bands. For these plots, $\Delta p_{\mathrm{sys}}^{+} \equiv p_{\mathrm{sys}}^{+}-p_{m}$ and $\Delta p_{\mathrm{sys}}^{-} \equiv p_{m}-p_{\mathrm{sys}}^{-}$.
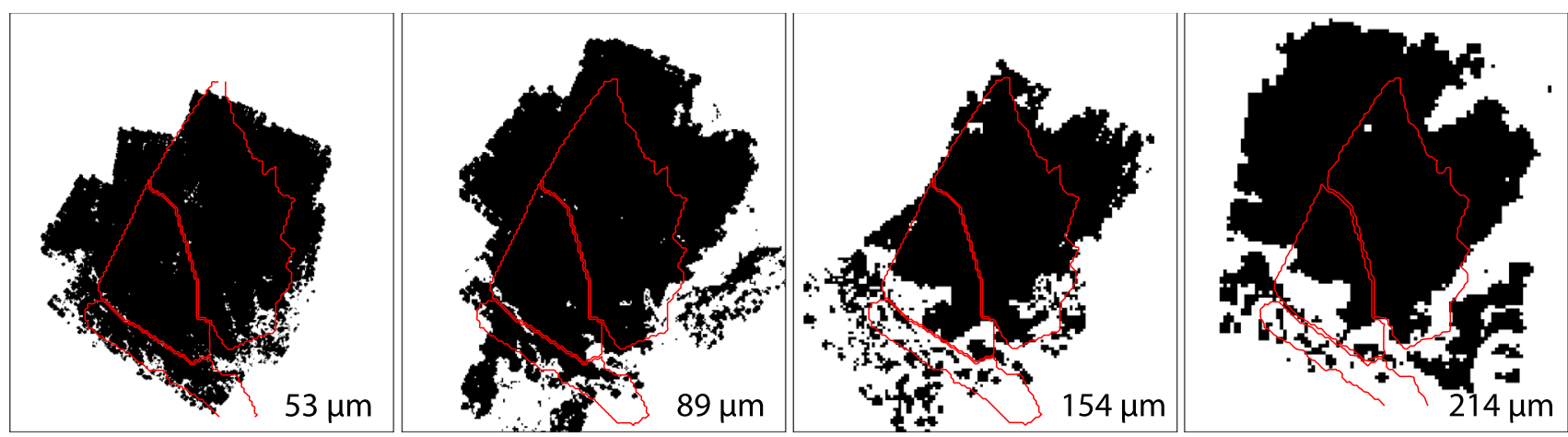

Figure 10. Pixel locations are shown (black) for the regions where the maximum effect of the reference beam affects the angles less than $10^{\circ}$. Region masks are overplotted for reference. The $3 \sigma$ data cut is also included.

\subsection{Fractional Polarization versus Intensity and Angle Dispersion}

The magnetic field in the interstellar medium (ISM) has both constant (threaded) and turbulent components (see a recent treatment by Planck Collaboration et al. 2018). The effects of a turbulent component can be seen in variations of the polarization angle with position on the sky, analyzed using angle dispersions (Davis 1951; Chandrasekhar \& Fermi 1953; Myers \& Goodman 1991; Pattle et al. 2017; Planck Collaboration et al. 2018) or a type of structure function (Kobulnicky et al. 1994; Hildebrand et al. 1999; Houde et al. 2016) and in the trend of fractional polarization with column density (e.g., Hildebrand et al. 1999; Jones \& Whittet 2015). We analyze the structure function in
Section 3.6, and in this section we examine the trend of fractional polarization with column density and angle dispersion.

If the magnetic field geometry is perfectly constant with no bends or wiggles, the fractional polarization in emission will be constant (Jones \& Whittet 2015, for a review) with column density. (For most lines of sight in our OMC-1 map, the farinfrared optical depth is $\ll 1$, so the effects of opacity on the polarization can be ignored.) If the magnetic field varies in a purely stochastic way along the line of sight on scales comparable to the beam, the fractional polarization will decrease as the square root of the column density (Jones \& Whittet 2015). A combination of a constant and a purely random component will cause the polarization to decrease with column density at a rate in between these two extremes. If there 

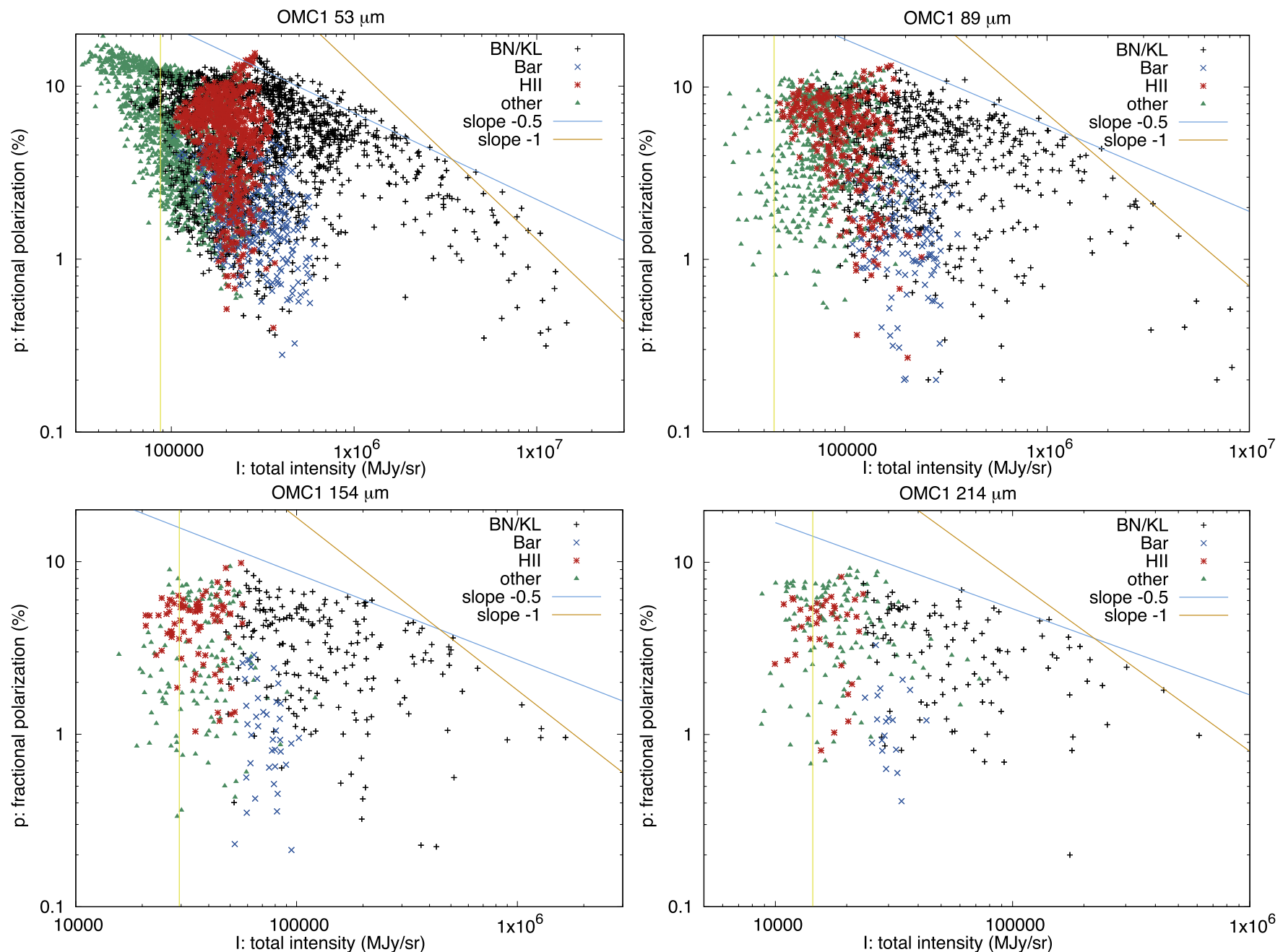

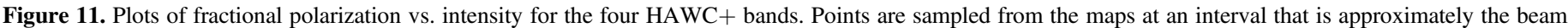

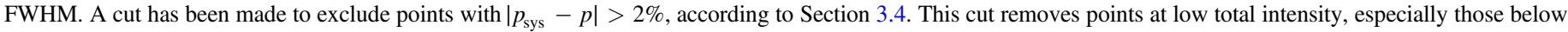

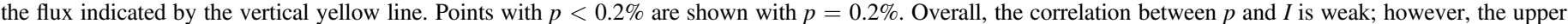

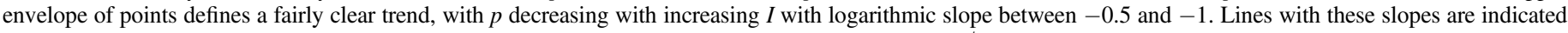
with diagonal lines in the plots. The points at high intensity in all four bands correspond to the BN/KL core region. See Section 3.5 for discussion.

is a coherent departure from a purely constant component such as a spiral twist, regions of mutually perpendicular fields, or a simple large-scale variation of the projected field along the line of sight, the fractional polarization can drop faster than the square root of the column density due to strong cancellation of the polarization. The Planck Collaboration et al. (2018) analysis of the polarized foreground in the Milky Way using both the structure function and the observed fractional polarization suggests this is common. In addition to cancellation effects reducing the fractional polarization, loss of grain alignment can cause dilution of the fractional polarization by unpolarized intensity from regions with unaligned grains (Andersson et al. 2015; Jones \& Whittet 2015).

The trend in fractional polarization with surface brightness (intensity) for all four bands is illustrated in Figure 11. We are concentrating on the upper bound in these plots because that delineates lines of sight where the minimum depolarization effects are present. If we roughly characterize the slope of the upper bound with a single power law $p \propto I^{\alpha}$ in each bandpass, we find $\alpha \sim-0.6$ to -0.7 . This is steeper than $p \propto I^{-1 / 2}$, indicating there must be large-scale variation of the projected field along the line of sight, loss of grain alignment for some fraction of the line of sight, or a combination of both. If the slope were $\alpha=-1$, often seen in dense protostellar cores (e.g., Galametz et al. 2018), then the denser regions would likely suffer loss of grain alignment (Jones \& Whittet 2015). Our result of a shallower slope, along with the fact that NIR polarization in extinction toward $\mathrm{BN}$ is the expected value for diffuse ISM extinction (Jones 1989; Poidevin et al. 2011), suggests loss of grain alignment cannot be the sole explanation for the decrease in fractional polarization with intensity. In fact, based on the evidence presented below, we conclude that the trend can be explained entirely by magnetic field structure, with no need to invoke variations in grain alignment.

As shown in Figure 11, the measured fractional polarization in OMC-1 ranges over a factor of 30 or more over the maps. There is a tendency for the largest fractional polarization $p$ to be found where the total intensity $I$ is relatively low and for the smallest $p$ to be found where $I$ is relatively high. However, overall the correlation of $p$ and $I$ is weak. A much better predictor of the fractional polarization along a particular line of sight is the dispersion of polarization angles in its vicinity, and in fact that is the strongest correlation we have found with $p$. The correlation of $p$ with angle dispersion $S$ was explored 

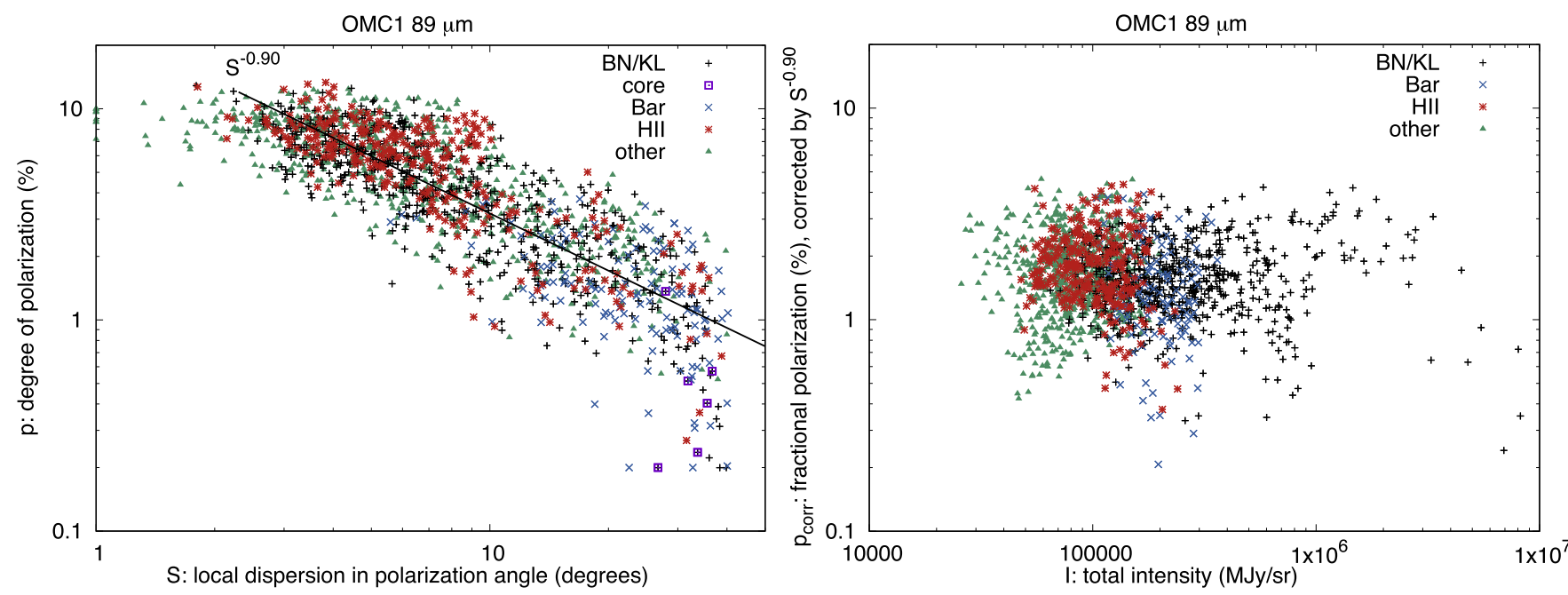

Figure 12. Left: fractional polarization vs. angle dispersion for OMC-1 at $89 \mu \mathrm{m}$, using $\theta_{S}=30^{\prime \prime}$. In addition to the $\left|p_{\text {sys }}-p\right|>2 \%$ cut, points have been limited to $p \geqslant 0.2 \%$ and $S \geqslant 1^{\circ}$. Points within the FWHM of the BN/KL I peak are highlighted (square "core" symbols); the polarization toward those lines of sight tends to fall below the trend. Right: fractional polarization vs. total intensity for OMC-1 at $89 \mu \mathrm{m}$, now with $p$ corrected for the angle dispersion trend by dividing each point by the local value of $(S / 12.5)^{-0.90}$. Again, the points at high intensity corresponding to the BN/KL core tend to fall below the trend.

Table 3

Fits to OMC-1 Polarization Trends

\begin{tabular}{lcl}
\hline \hline $\begin{array}{l}\text { Wavelength } \\
(\mu \mathrm{m})\end{array}$ & $\begin{array}{c}\theta_{S} \\
(\operatorname{arcsec})\end{array}$ & Best-fit Trend \\
\hline 53 & 30 & $p \approx 3.1 \%\left(I / 3.8 \times 10^{5} \mathrm{MJy} \mathrm{sr}^{-1}\right)^{-0.01}\left(S / 14^{\circ} .2\right)^{-0.87}$ \\
89 & 30 & $p \approx 2.6 \%\left(I / 2.4 \times 10^{5} \mathrm{MJy} \mathrm{sr}^{-1}\right)^{-0.09}(S / 12.5)^{-0.90}$ \\
154 & 30 & $p \approx 1.9 \%\left(I / 1.0 \times 10^{5} \mathrm{MJy} \mathrm{sr}^{-1}\right)^{-0.19}\left(S / 12^{\circ} 5\right)^{-0.84}$ \\
214 & 30 & $p \approx 2.3 \%\left(I / 0.42 \times 10^{5} \mathrm{MJy} \mathrm{sr}^{-1}\right)^{-0.21}(S / 8.6)^{-0.70}$ \\
\hline
\end{tabular}

previously for Planck and BLASTPol submillimeter polarimetry at $\geqslant 5^{\prime}$ scales (Planck Collaboration et al. 2015, 2018; Fissel et al. 2016). Those authors found trends with $p \propto S^{-0.6}$ to $S^{-1}$. Planck Collaboration et al. (2018) developed a model for turbulent magnetic field structure predicting $p \propto S^{-1}$.

Here we use a simple method of calculating the dispersion of polarization angles: for a given line of sight, we compute the rms of the angles within a diameter of $\theta_{S}$ centered on that line of sight. We exclude from the calculation angle measurements with statistical uncertainty $>10^{\circ}$. We use Stokes parameters to avoid the complication of the branch of position angles. In detail,

$$
\begin{aligned}
S & =\sqrt{\left\langle(\phi-\bar{\phi})^{2}\right\rangle} \approx \sqrt{\left\langle\sin ^{2}(\phi-\bar{\phi})\right\rangle} \\
& =\sqrt{(1-\langle\hat{q}\rangle \hat{q}(\bar{\phi})-\langle\hat{u}\rangle \hat{u}(\bar{\phi})) / 2},
\end{aligned}
$$

in radians, computed within a circular region centered on a given line of sight (associated with a particular measurement of p), where

$$
\hat{q}_{i}=\cos 2 \phi_{i}, \hat{u_{i}}=\sin 2 \phi_{i} .
$$

To remove approximately the effect of noise bias on the measurements of $S$, we debias the results by subtracting the rms uncertainty in $\phi$ over the aperture: $S_{\text {debiased }}=\sqrt{S^{2}-\sigma^{2}(\phi)}$. This is a small effect over most of these OMC-1 maps. In the rest of the paper, we use $S$ as shorthand for $S_{\text {debiased. }}$.

Figure 12 shows the observed relationship between $S$ and $p$ for the $89 \mu \mathrm{m}$ observations of OMC-1. Following Fissel et al. (2016), we fit the trend in $p$ as a function of two parameters, $S$ and total intensity $I$ :

$$
p \approx p_{0}(I / \bar{I})^{\alpha_{I}}(S / \bar{S})^{\alpha_{S}} .
$$

The fit results are shown for all bands in Table 3 and for $89 \mu \mathrm{m}$ in Figure 12. For this initial look at the far-infrared angle dispersion, we used a common value of $\theta_{S}=30^{\prime \prime}$ for all bands, just large enough to allow calculation of $S$ at $214 \mu \mathrm{m}$.

Our fit to the data finds a dependence $p \propto S^{-0.9}$ to $S^{-0.7}$, close to the $S^{-1}$ form expected for a simple model of magnetic field structure (Appendix E by Planck Collaboration et al. 2018). With that dependence removed, there is little further dependence on $I$ for OMC-1: fits for the power-law index $\alpha_{I}$ range from -0.01 to -0.21 . Standard error propagation indicates uncertainty $\sigma\left(\alpha_{I}\right)$ of $\sim 0.01$ at $53 \mu \mathrm{m}$ increasing to $\sim 0.03$ at $214 \mu \mathrm{m}$ due to the smaller number of measurements; however, the $\chi^{2}$ for the polarization model increases by only $\lesssim 30 \%$ if the $I^{\alpha_{I}}$ term is omitted. On the other hand, Fissel et al. (2016) found a clear $I^{-0.45}$ dependence in their BLASTPol $500 \mu \mathrm{m}$ observations of Vela C (along with $S^{-0.60}$ ) with loss of grain alignment in denser regions offered as a possible explanation. In OMC-1, we do not see clear evidence for poorer grain alignment in dense regions up to column densities of $N_{\mathrm{H}} \approx 10^{23} \mathrm{~cm}^{-2}$, as further demonstrated qualitatively by Figure 7. To first approximation, the observed distribution of fractional polarization can be explained by the magnetic field structure of the cloud. OMC-1 has a stronger radiation field from its embedded stars than does Vela $C$, which in the context of RATs (B-RAT, in this case) (Lazarian \& Hoang 2007) could 
explain the difference between our results and those of Fissel et al. (2016). To make a rough estimate of the difference in intensity of the radiation field, we use the dust temperature, which has a median value of $36 \mathrm{~K}$ for OMC-1 (Section 3.1) and median value of $\sim 15 \mathrm{~K}$ for Vela $\mathrm{C}$ (Hill et al. 2011). This corresponds to a ratio of $(36 / 15)^{4} \approx 30$ in intensities.

Especially at 53 and $89 \mu \mathrm{m}$, the fractional polarization toward the BN/KL core falls below the trend with $I$ and $S$ (Figures 11 and 12). Schleuning (1998) argued that low $100 \mu \mathrm{m}$ polarization toward $\mathrm{BN} / \mathrm{KL}$ is due to optical depth $\approx 0.6$. Such a value corresponds to $\sim 4 \times 10^{23} \mathrm{~cm}^{-2}$ in Figure 3 and is localized to the two peaks along the ridge. The suppression of polarization due to optical depth should be greater at shorter wavelengths, and in fact the $\mathrm{HAWC}+$ data show a monotonic trend of fractional polarization decreasing with decreasing wavelength. In a $30^{\prime \prime}$ diameter aperture centered on BN/KL (matching Schleuning 1998), the fractional polarization is $0.44 \%, 0.71 \%, 1.10 \%$, and $1.47 \%$ at 53 , 89,154 , and $214 \mu \mathrm{m}$, respectively. Further supporting the hypothesis of optical depth significantly influencing the fractional polarization is the SED of BN/KL (Section 3.1). The calculated $53 \mu \mathrm{m}$ optical depth toward BN/KL, at 18 !! 7 resolution, is 0.8 ; this optical depth reduces the emergent fractional polarization by a factor of $\sim 1.5$ at $53 \mu \mathrm{m}$ and less at longer wavelengths (Dowell 1997) - insufficient to fully explain the trend with wavelength. However, we note that there is clear wavelength-dependent polarization angle structure within the $30^{\prime \prime}$ aperture (Figures 6 and 7) that could also play a role in the variation of polarization fraction, and we also have not considered the clumpiness of the emitting medium.

The maximum fractional polarization, corresponding to favorable conditions of field orientation and order and of grain alignment, provides a lower limit to the elongation of dust grains (Hildebrand \& Dragovan 1995; Draine \& Hensley 2017; Guillet et al. 2018). In the HAWC+ maps of OMC-1, one local maximum in the fractional polarization is seen most clearly at $89 \mu \mathrm{m}$, located at $\alpha_{\mathrm{J} 2000}=5^{\mathrm{h}} 35^{\mathrm{m}} 21^{\mathrm{s}} \delta_{\mathrm{J} 2000}=-5^{\circ} 21^{\prime} 50^{\prime \prime}$ in an elongated polarized flux feature that does not correspond closely to a feature in total intensity. The maximum observed fractional polarization is $14.0 \%$, and the total intensity is $4 \mathrm{Jy} \operatorname{arcsec}^{-2}$. The Herschel $100 \mu \mathrm{m}$ map indicates a sourceto-reference beam intensity ratio of approximately 25 for this line of sight and SOFIA chop (Section 2); the minimum intrinsic polarization that could produce $14.0 \%$ observed polarization (via perpendicular source and reference beam polarization angles) is $12.9 \%$. The same feature has an observed fractional polarization of $15.4 \%, 9.3 \%$, and $8.2 \%$ at 53, 154, and $214 \mu \mathrm{m}$, respectively. Other local maxima with higher fractional $89 \mu \mathrm{m}$ polarization are located where the intensity is far less, making those measurements vulnerable to significant reference beam effects, so they are not considered further.

Ward-Thompson et al. (2017) have noted a coherent magnetic field structure with high fractional $850 \mu \mathrm{m}$ polarization in a filament northeast of BN/KL. Only our $214 \mu \mathrm{m}$ map has good coverage of this feature. It is clearly defined in polarized intensity and has a maximum fractional polarization of $9.6 \%$ toward $\alpha_{\mathrm{J} 2000}=5^{\mathrm{h}} 35^{\mathrm{m}} 22^{\mathrm{s}} \delta_{\mathrm{J} 2000}=-5^{\circ} 19^{\prime} 50^{\prime \prime}$, where the total intensity is $0.6 \mathrm{Jy} \operatorname{arcsec}^{-2}$, approximately $10 \times$ the reference beam flux. The minimum intrinsic polarization that could produce this is $7.9 \%$.
In summary, our observations of OMC-1 have identified lines of sight with fractional polarization $\geqslant 14 \%, \geqslant 13 \%$, and $\geqslant 8 \%$ at 53,89 , and $214 \mu \mathrm{m}$, respectively. At the shorter wavelengths, this is somewhat higher than the maximum of $9 \%$ found by Hildebrand \& Dragovan (1995) at $100 \mu \mathrm{m}$; with further HAWC + observations of OMC-1 or other fields, one may find still higher polarization in the far-infrared. At slightly longer wavelengths, a maximum fractional polarization of $8 \%-$ $13 \%$ was observed in the Vela $\mathrm{C}$ cloud, mapped over a $\sim 1^{\circ}$ area with $5^{\prime}$ resolution at $\lambda=250-500 \mu \mathrm{m}$ (Gandilo et al. 2016). At $850 \mu \mathrm{m}$, a maximum dust fractional polarization of $\sim 22 \%$ has been measured elsewhere in the Galaxy (Benoît et al. 2004; Planck Collaboration et al. 2018); the sensitivity to relatively diffuse clouds is a likely explanation for the higher value compared to the OMC-1 data we are reporting here.

\subsection{Magnetic Field Strength}

The DCF method (Davis 1951; Chandrasekhar \& Fermi 1953) can be used to obtain estimates of the plane-ofthe-sky magnetic field strength by comparing the dispersion of polarization vectors to the velocity dispersion. One challenge with this technique is that the large-scale field structure can contribute to the dispersion. In order to separate the dispersion due to the turbulent-field component from that of the largescale field, an isotropic two-point structure function, or dispersion function (DF), can be calculated to characterize the dispersion as a function of angular scale (Hildebrand et al. 2009; Houde et al. 2009, 2011, 2016). The DF can be fit with a model that separates the large-scale contribution from that of the turbulence (Houde et al. 2016),

$$
\begin{aligned}
& 1-\langle\cos [\Delta \phi(l)]\rangle \\
& =\frac{1}{1+\mathcal{N}\left[\frac{\left\langle B_{t}^{2}\right\rangle}{\left\langle B_{0}^{2}\right\rangle}\right]^{-1}}\left\{1-\exp \left(-\frac{l^{2}}{2\left(\delta^{2}+2 W^{2}\right)}\right)\right\}+a_{2} l^{2},
\end{aligned}
$$

where the first term accounts for the small-scale turbulent contribution to the dispersion (taking into account the correlations due to beam size) and the second term corresponds to the ordered, large-scale field contribution. In Equation (12), $l$ is the distance between a pair of vectors with angle difference $\Delta \phi$, and $W$ corresponds to the beam radius. Angle brackets indicate average values, $\frac{\left\langle B_{t}^{2}\right\rangle}{\left\langle B_{0}^{2}\right\rangle}$ is the turbulent-to-ordered field ratio, and $\mathcal{N}$ is the number of turbulent cells in the gas column given by Houde et al. (2009)

$$
\mathcal{N}=\frac{\left(\delta^{2}+2 W^{2}\right) \Delta^{\prime}}{\sqrt{2 \pi} \delta^{3}}
$$

In Equations (12) and (13), $\delta$ is the correlation length for the turbulent field, and $\Delta^{\prime}$ is the effective thickness of the cloud. See Houde et al. $(2009,2011,2016)$ for full details on the model above. In fitting the DFs using the equations above, there are four parameter to be determined: $\frac{\left\langle B_{t}^{2}\right\rangle}{\left\langle B_{0}^{2}\right\rangle}, \delta, a_{2}$, and $\Delta^{\prime}$. The parameters $\frac{\left\langle B_{t}^{2}\right\rangle}{\left\langle B_{0}^{2}\right\rangle}$ and $\Delta^{\prime}$ are highly degenerate, so we fit for $a_{2}, \delta$, and $\Delta^{\prime \prime} \equiv \Delta^{\prime}\left(\frac{\left\langle B_{t}^{2}\right\rangle}{\left\langle B_{0}^{2}\right\rangle}\right)^{-1}$. Following Houde et al. (2009), $\Delta^{\prime}$ 
(a) HAWC $+53 \mu \mathrm{m}$
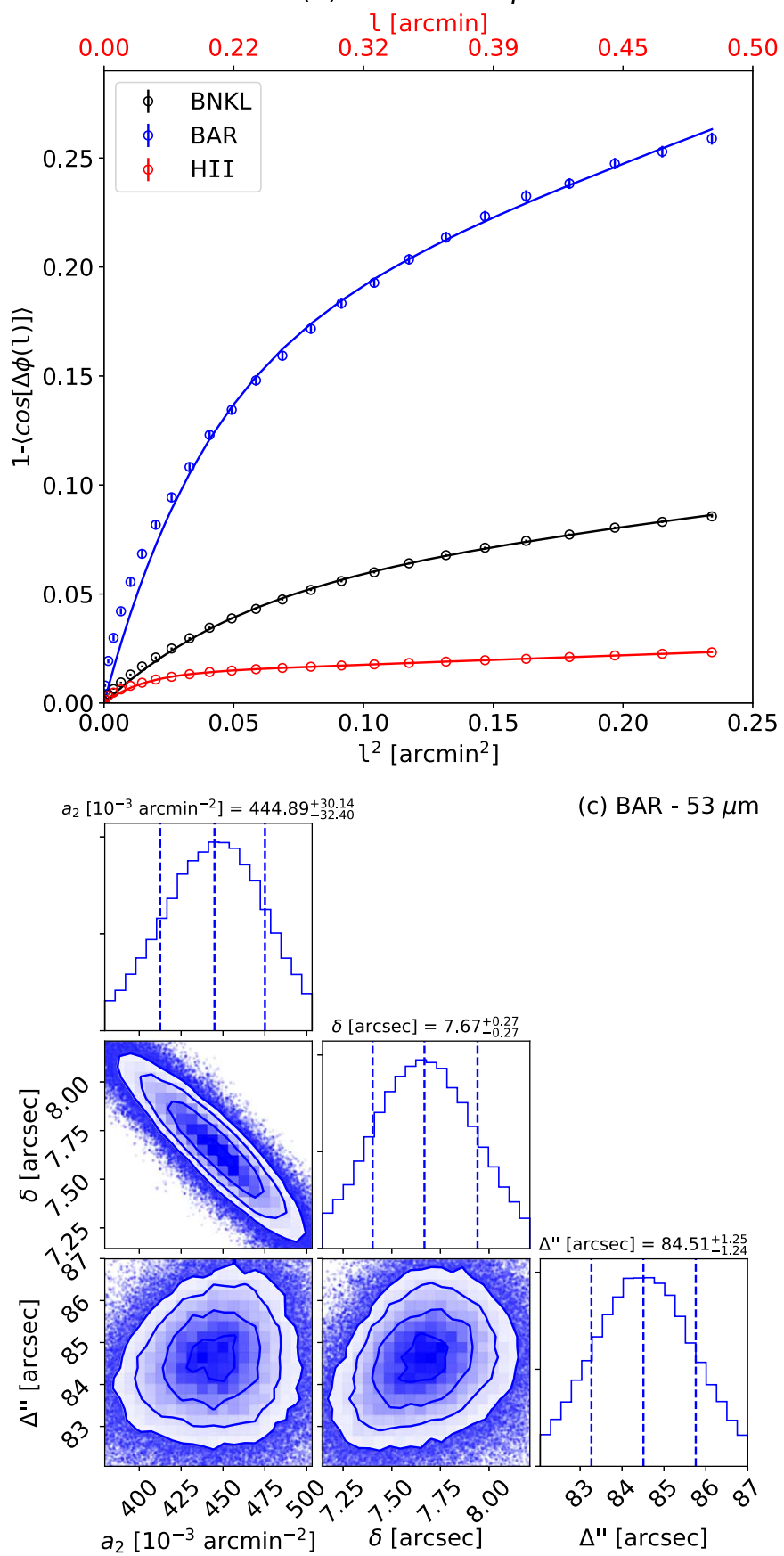
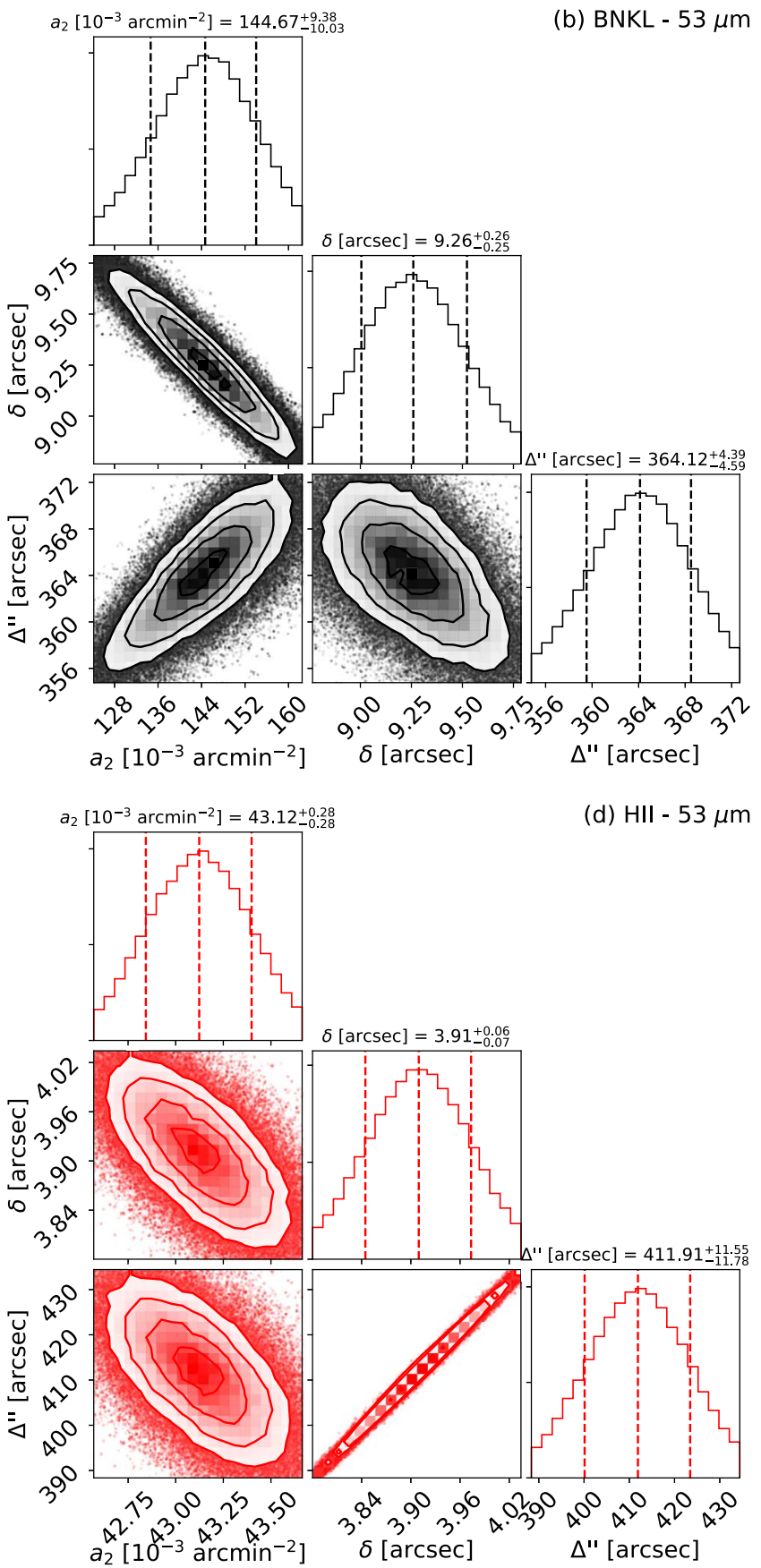

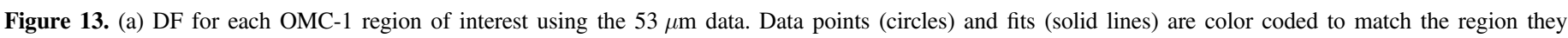

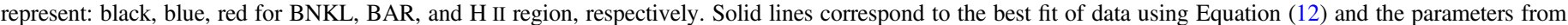

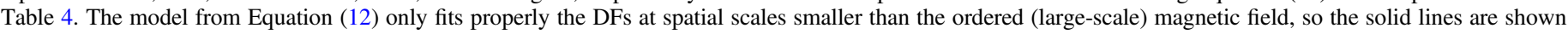
only over the $l$-values used in each fit. (b)-(d) Results from the MCMC solver for each studied region.

can be estimated by the FWHM value of the isotropic autocorrelation of the polarized intensity.

We implemented an MCMC solver for fitting the nonlinear model of Equation (12) to the DFs and determining the optimal model parameters and their associated uncertainties. The model represented by Equation (12) is only valid at small values of $l$ $(\approx 0$ ' $1-0$ ' 5 up to $\sim 5-7$ times the size of the beam; Houde et al. 2009, 2016). First, a preliminary solution is found by running the MCMC algorithm using uncertainties calculated according to Houde et al. (2016). Final solutions are found by repeating the MCMC process and inflating the errors by the square root of the reduced goodness-of-fit coefficient, $\chi_{r}^{2}$.

In Figure 13(a) we present the DF (circles) for $53 \mu \mathrm{m}$ data and best fit (solid lines) for small scales $(l \lesssim 0.5$ arcmin). Data points and lines are color coded to match the regions in Figure 8: black, blue, and red for BNKL, BAR, and H II, respectively. As expected, all three curves show a dispersion that increases nonlinearly with angular distance $l$. As shown in 
Table 4

Parameters for the OMC-1 Regions BNKL, BAR, and H II, Derived from the Analysis of Polarization Vectors Dispersion

\begin{tabular}{|c|c|c|c|}
\hline $\begin{array}{l}\text { Wavelength } \\
(\mu \mathrm{m})\end{array}$ & $\begin{array}{c}a_{2} \\
\left(10^{-3} \operatorname{arcmin}^{-2}\right)\end{array}$ & $\begin{array}{c}\delta \\
(\operatorname{arcsec})\end{array}$ & $\begin{array}{c}\Delta^{\prime \prime} \\
(\operatorname{arcsec})\end{array}$ \\
\hline \multicolumn{4}{|l|}{ BNKL } \\
\hline 53 & $144.67_{-10.03}^{+9.38}$ & $9.26_{-0.25}^{+0.26}$ & $364.12_{-4.59}^{+4.39}$ \\
\hline 89 & $74.34_{-1.95}^{+1.92}$ & $10.26_{-0.30}^{+0.30}$ & $387.36_{-9.85}^{+9.41}$ \\
\hline 154 & $36.02_{-3.70}^{+3.34}$ & $21.69_{-1.67}^{+1.79}$ & $622.90_{-22.55}^{+23.16}$ \\
\hline 214 & $7.15_{-3.47}^{+3.25}$ & $33.85_{-2.61}^{+2.68}$ & $707.10_{-30.76}^{+30.59}$ \\
\hline \multicolumn{4}{|l|}{ BAR } \\
\hline 53 & $444.89_{-32.40}^{+30.14}$ & $7.67_{-0.27}^{+0.27}$ & $84.51_{-1.24}^{+1.25}$ \\
\hline 89 & $158.65_{-8.69}^{+8.59}$ & $10.24_{-0.20}^{+0.21}$ & $94.74_{-1.09}^{+1.08}$ \\
\hline 154 & $\ldots$ & $\ldots$ & $\ldots$ \\
\hline 214 & $\ldots$ & $\ldots$ & $\ldots$ \\
\hline \multicolumn{4}{|l|}{ H II } \\
\hline 53 & $43.12_{-0.28}^{+0.28}$ & $3.91_{-0.07}^{+0.06}$ & $411.91_{-11.78}^{+11.5}$ \\
\hline 89 & $18.85_{-1.55}^{+1.48}$ & $9.29_{-0.57}^{+0.57}$ & $744.77_{-50.14}^{+46.93}$ \\
\hline 154 & $12.60_{-0.73}^{+0.73}$ & $9.37_{-0.59}^{+0.42}$ & $941.24_{-95.68}^{+43.67}$ \\
\hline 214 & $14.78_{-0.74}^{+0.73}$ & $10.20_{-1.09}^{+0.61}$ & $888.12_{-174.00}^{+82.86}$ \\
\hline
\end{tabular}

Note. These parameters were obtained by means of an MCMC solver fitting the model in Equation (12) for the DFs. Parameter values correspond to the quartile 0.5 (median), while errors correspond to the percentiles 0.16 and 0.84 .

Figure 13(a), the highest level of dispersion is present in the OMC-1 bar. The BNKL region, in turn, shows a lower level of dispersion than BAR but higher than that of $\mathrm{H}$ II, which has the lowest level of dispersion of the three regions. This observation indicates the presence of a larger turbulent-field component in the BAR region than in the other two regions, which is in qualitative agreement with the visual inspection of the region in Figure 6. However, if the explanation for the low observed fractional polarization is the superposition and cancellation of large-scale fields, the dispersion and ultimately $\frac{\left\langle B_{t}^{2}\right\rangle}{\left\langle B_{0}^{2}\right\rangle}$ may be overestimated by this technique.

Final best-fit parameters are summarized in Table 4. These results show clear differences among the three OMC-1 regions. Contributions in all regions to the dispersion from the ordered term $a_{2}$ seem to decrease with increasing wavelength. When comparing $a_{2}$ in the different regions, the values for H II and BNKL are up to one order of magnitude lower than those in BAR. On the other hand, when examining the values of $\delta$ and $\Delta^{\prime}$, we observe that in BNKL and BAR, these parameters increase with increasing wavelength, possibly in a nonlinear way because the values for 53 and $89 \mu \mathrm{m}$ are more similar to each other than to those of 154 and $214 \mu \mathrm{m}$ values. For the H II region, parameters $\delta$ and $\Delta^{\prime}$ seem to increase with increasing wavelength (within their errors). This may be indicative of the presence of dust at different temperatures along the line of sight in BNKL and BAR.

Following Houde et al. (2009), the parameters that characterize the turbulence in the studied regions, $\mathcal{N}$ and $\frac{\left\langle B_{t}^{2}\right\rangle}{\left\langle B_{0}^{2}\right\rangle}$, can be calculated as

$$
\mathcal{N}(53 \mu \mathrm{m})=6.67\left(\frac{\Delta^{\prime}}{134 \operatorname{arcsec}}\right)
$$

and

$$
\frac{\left\langle B_{t}^{2}\right\rangle}{\left\langle B_{0}^{2}\right\rangle}(53 \mu \mathrm{m})=\frac{\Delta^{\prime}}{\Delta^{\prime \prime}}=0.37\left(\frac{\Delta^{\prime}}{134 \operatorname{arcsec}}\right) .
$$

In the equations above we have used $\delta$ and $W$ for BNKL $53 \mu \mathrm{m}$ data from Tables 4 and 1 . As mentioned above, $\Delta^{\prime}$ can be calculated as described in Houde et al. (2009). We found $\Delta^{\prime}=2.27,2.80,3.80$, and 4.97 arcmin for 53, 89, 154, and $214 \mu \mathrm{m}$ data, respectively. These values, were obtained using the entire field of view in each band. Consequently, the strength of the large-scale magnetic field can be calculated as

$$
B_{0} \simeq \sqrt{4 \pi \rho} \sigma(v)\left[\frac{\left\langle B_{t}^{2}\right\rangle}{\left\langle B_{0}^{2}\right\rangle}\right]^{-1 / 2}=\sqrt{4 \pi \rho} \sigma(v)\left[\frac{\Delta^{\prime}}{\Delta^{\prime \prime}}\right]^{-1 / 2},
$$

which is a modified version of the DCF relation. Using the fitted parameters for BNKL $53 \mu \mathrm{m}$, this leads to an estimate of the magnetic field strength in this region.

$$
\begin{aligned}
B_{0}(53 \mu \mathrm{m})= & 1002\left(\frac{\mathrm{N}\left(\mathrm{H}_{2}\right)}{9.85 \times 10^{22} \mathrm{~cm}^{-2}}\right)^{1 / 2} \\
& \times\left(\frac{L}{4.34 \times 10^{17} \mathrm{~cm}}\right)^{-1 / 2} \\
& \times\left(\frac{\sigma(v)}{1.85 \times 10^{5} \mathrm{~cm} \mathrm{~s}^{-1}}\right)\left(\frac{\Delta^{\prime}}{134 \operatorname{arsec}}\right)^{-1 / 2} \mu \mathrm{G} .
\end{aligned}
$$

Here we have applied a nominal velocity dispersion value $\sigma$ $(v)=1.85 \mathrm{~km} \mathrm{~s}^{-1}$ (Houde et al. 2009) for all OMC-1 regions. Column densities correspond to average values for each region in the $\mathrm{N}\left(\mathrm{H}_{2}\right)$ map of Figure 3. In order to transform column density to mass density we assume a uniform cloud depth $L=4.34 \times 10^{17} \mathrm{~cm}$ (Pattle et al. 2017). Resulting values for $\frac{\left\langle B_{t}^{2}\right\rangle}{\left\langle B_{0}^{2}\right\rangle}, B_{0}$, and $\mathcal{N}$ for all regions and bands are presented in Table 5. Due to the potential contamination by reference intensity in some parts of the BAR region, only a very low number of pixels can be used for the analysis at 154 and $214 \mu \mathrm{m}$ (Figure 10), so DFs for the BAR were not calculated in these bands. The results in Table 4 show the BNKL region having the strongest magnetic field strength, $\sim 0.9-1.0 \mathrm{mG}$. The estimates for magnetic field strength in regions $\mathrm{H}$ II and BAR are approximately one-third of the BNKL values, $\sim 300 \mu \mathrm{G}$. These values of plane-of-the-sky magnetic field strength are similar to the average value of $760 \mu \mathrm{G}$ for the entire OMC-1 region estimated by Houde et al. (2009) but significantly below the $6.6 \pm 4.7 \mathrm{mG}$ estimated by Pattle et al. (2017). Our results indicate variation of magnetic field structure, not only in geometry but also in strength, across OMC-1. Consequently, it is possible that the magnetic field strength in OMC-1 displays significant spatial variations within each studied region, which, in the case of BNKL, can play a significant role in energy balance and magnetic dominance of the explosion observed in $\mathrm{H}_{2}$ and $\mathrm{CO}$ emissions (See Section 3.7).

Our estimates of $\frac{\left\langle B_{t}^{2}\right\rangle}{\left\langle B_{0}^{2}\right\rangle}$ show that within the regions of the OMC-1 cloud studied here, H II and BAR are extreme cases in terms of turbulent states. The $\mathrm{H}$ II region seems to be a more ordered region with small turbulent components (turbulent field 0.23-0.34 times $\left\langle B_{0}^{2}\right\rangle$ ). The BAR, in contrast, appears as a 
Table 5

Physical Parameters for OMC-1 Regions Derived from the Results of the Dispersion Analysis

\begin{tabular}{|c|c|c|c|c|}
\hline $\begin{array}{l}\text { Wavelength } \\
(\mu \mathrm{m})\end{array}$ & $\begin{array}{l}\mathrm{N}\left(\mathrm{H}_{2}\right) \\
\left(\mathrm{cm}^{-2}\right)\end{array}$ & $\frac{\left\langle B_{t}^{2}\right\rangle}{\left\langle B_{0}^{2}\right\rangle}$ & $\begin{array}{c}B_{0} \\
(\mu \mathrm{G})\end{array}$ & $\mathcal{N}$ \\
\hline \multicolumn{5}{|l|}{ BNKL } \\
\hline 53 & $(9.85 \pm 8.96) \times 10^{22}$ & 0.37 & 1002 & 6.67 \\
\hline 89 & $\ldots$ & 0.43 & 931 & 8.42 \\
\hline 154 & $\cdots$ & 0.37 & 1013 & 5.02 \\
\hline 214 & $\cdots$ & 0.42 & 944 & 4.02 \\
\hline \multicolumn{5}{|l|}{ BAR } \\
\hline 53 & $(3.87 \pm 2.12) \times 10^{22}$ & 1.61 & 303 & 8.50 \\
\hline 89 & $\ldots$ & 1.77 & 289 & 8.44 \\
\hline 154 & $\cdots$ & $\ldots$ & $\ldots$ & \\
\hline 214 & $\cdots$ & $\cdots$ & $\cdots$ & \\
\hline \multicolumn{5}{|l|}{ H II } \\
\hline 53 & $(5.90 \pm 3.24) \times 10^{21}$ & 0.33 & 261 & 24.59 \\
\hline 89 & $\ldots$ & 0.23 & 316 & 9.76 \\
\hline 154 & $\cdots$ & 0.24 & 305 & 19.32 \\
\hline 214 & $\cdots$ & 0.34 & 259 & 30.23 \\
\hline
\end{tabular}

Note. For each region/band the following parameters are reported: (a) $\mathrm{N}\left(\mathrm{H}_{2}\right)$, average column density of molecular hydrogen; (b) turbulent-to-large-scale field ratio; (c) $B_{0}$, plane-of-the-sky magnetic field intensity; (d) $\mathcal{N}$, number of turbulent cells in the gas column. The uncertainties reported in the table are dominated by the uncertainty in the cloud depth (see Equations (14)-(16)), which could be uncertain by a factor of $\sim 2$.

highly turbulent region with components even greater than the large-scale field $\left(\frac{\left\langle B_{t}^{2}\right\rangle}{\left\langle B_{0}^{2}\right\rangle}>1\right)$. However, as discussed in Section 3.2, the low polarization in the bar may indicate variations in grain alignment, a field predominantly oriented along the line of sight, a superposition of canceling (orthogonal) fields, or a combination of these effects. Such considerations would lower the value of the inferred large-scale field and systematically inflate the dynamical importance of the turbulence. BNKL lies between these two regimes-it shows between 0.37 and 0.43 times $\left\langle B_{0}^{2}\right\rangle$ for the turbulent component. In terms of the number of turbulent cells present in the gas column, BNKL presents the lowest $\mathcal{N}(\approx 5-8)$, while the H II region shows the highest $\mathcal{N}(\approx 10-30)$.

\subsection{The BNKL Explosion}

The BNKL region has been identified as a site of a massive explosion possibly powered by stellar interactions (Bally \& Zinnecker 2005; Bally et al. 2011). The energy associated with this explosion has been estimated to be of the order $\sim 10^{47}$ erg (Snell et al. 1984). The center of the explosion, which is roughly centered on the peak of the $53 \mu \mathrm{m}$ intensity, is traced by high-velocity CO emission "fingertips" out to $30^{\prime \prime}-45^{\prime \prime}$ (Bally et al. 2017). These are distributed nearly isotropically in the plane of the sky around the center of the explosion. Farther out, the explosion is traced by a bipolar outflow of $\mathrm{H}_{2}$ "fingers" that extend $2^{\prime}-3^{\prime}$ to the north-northwest and $2^{\prime}$ to the southsoutheast. The field lines inferred from the $53 \mu \mathrm{m} \mathrm{HAWC}+$ observations appear to trace the $\mathrm{H}_{2}$ fingers; however, they do not trace the isotropic pattern of the high-velocity $\mathrm{CO}$, as shown in Figure 14. This suggests two possibilities for the magnetodynamics in the region. First, the magnetic field could be confining the flow, shaping the bipolar feature by allowing the explosion to expand preferentially parallel to the large-scale field. Second, the field could be being dragged by the explosion.

It is possible to get a sense for the critical value of the field (i.e., that associated with the required energy needed to shape the outflow) based on energy considerations. Because the field traces the bipolar pattern of the explosion in the larger volume defined by the $\mathrm{H}_{2}$ fingers, but not in the smaller volume of the $\mathrm{CO}$ emission, we calculate the critical value of the field in each of these regions. For the smaller region, where the $\mathrm{CO}$ streams dominate, we assume a sphere of angular radius $\theta \sim 30^{\prime \prime}$. In this case, the mean energy density in the explosion can be approximated by

$$
\begin{aligned}
u_{\text {explosion }}= & 8.3 \times 10^{-6}\left(\frac{D}{400 \mathrm{pc}}\right)^{-3}\left(\frac{\theta}{30^{\prime \prime}}\right)^{-3} \\
& \times\left(\frac{E}{2 \times 10^{47} \mathrm{erg}}\right) \operatorname{erg~\mathrm {cm}^{-3}}
\end{aligned}
$$

Here $D$ is the distance to BNKL and $E$ is the total energy of the explosion. The magnetic field is given by $B=\sqrt{8 \pi u_{M}}$, where $u_{M}$ is the magnetic energy density. We can define a mean critical field, $B_{\text {crit }}$, as that required to produce magnetic energy density that is equal to the kinetic by setting $u_{M}=u_{\text {explosion, }}$

$$
\begin{aligned}
B_{\text {crit }}= & \sqrt{8 \pi u_{M}}=14.4\left(\frac{D}{400 \mathrm{pc}}\right)^{-3 / 2}\left(\frac{\theta}{30^{\prime \prime}}\right)^{-3 / 2} \\
& \times\left(\frac{E}{2 \times 10^{47} \mathrm{erg}}\right)^{1 / 2} \mathrm{mG} .
\end{aligned}
$$

For the larger volume, we assume a cylindrical volume of radius $\theta_{R} \sim 30^{\prime \prime}$ and height $\theta_{\mathrm{H}} \sim 230^{\prime \prime}$. We assume that the total energy in this volume is $\sim 1 \%$ of that of the explosion itself. We calculate a similar critical field as above:

$$
\begin{aligned}
u_{\text {explosion }}= & 1.4 \times 10^{-8}\left(\frac{D}{400 \mathrm{pc}}\right)^{-3}\left(\frac{\theta_{R}}{30^{\prime \prime}}\right)^{-2}\left(\frac{\theta_{\mathrm{H}}}{230^{\prime \prime}}\right)^{-1} \\
& \times\left(\frac{E}{2 \times 10^{45} \mathrm{erg}}\right) \mathrm{erg} \mathrm{cm}^{-3} .
\end{aligned}
$$

The value of the mean critical magnetic field is then

$$
\begin{aligned}
B_{\text {crit }}= & 0.6\left(\frac{D}{400 \mathrm{pc}}\right)^{-3 / 2}\left(\frac{\theta_{R}}{30^{\prime \prime}}\right)^{-1}\left(\frac{\theta_{\mathrm{H}}}{230^{\prime \prime}}\right)^{-1 / 2} \\
& \times\left(\frac{E}{2 \times 10^{45} \mathrm{erg}}\right)^{1 / 2} \mathrm{mG} .
\end{aligned}
$$

Given the values for the BNKL region found in Section 3.6 from the dispersion, we tentatively conclude that in the central $0.1 \mathrm{pc}$, the dynamics are dominated by the explosion as the magnetic field is much smaller than that required for energy balance with the explosion $(\sim 14 \mathrm{mG})$. This may explain why the high-velocity $\mathrm{CO}$ gas shows an approximately isotropic distribution in the plane of the sky. On larger scales, the magnetic field strength estimates are $\sim 0.9-1.0 \mathrm{mG}$, which is in the range of field strength for which the magnetic energy density is of the same order of magnitude as that for the explosion $(\sim 0.6 \mathrm{mG})$. This could explain the common bipolar 

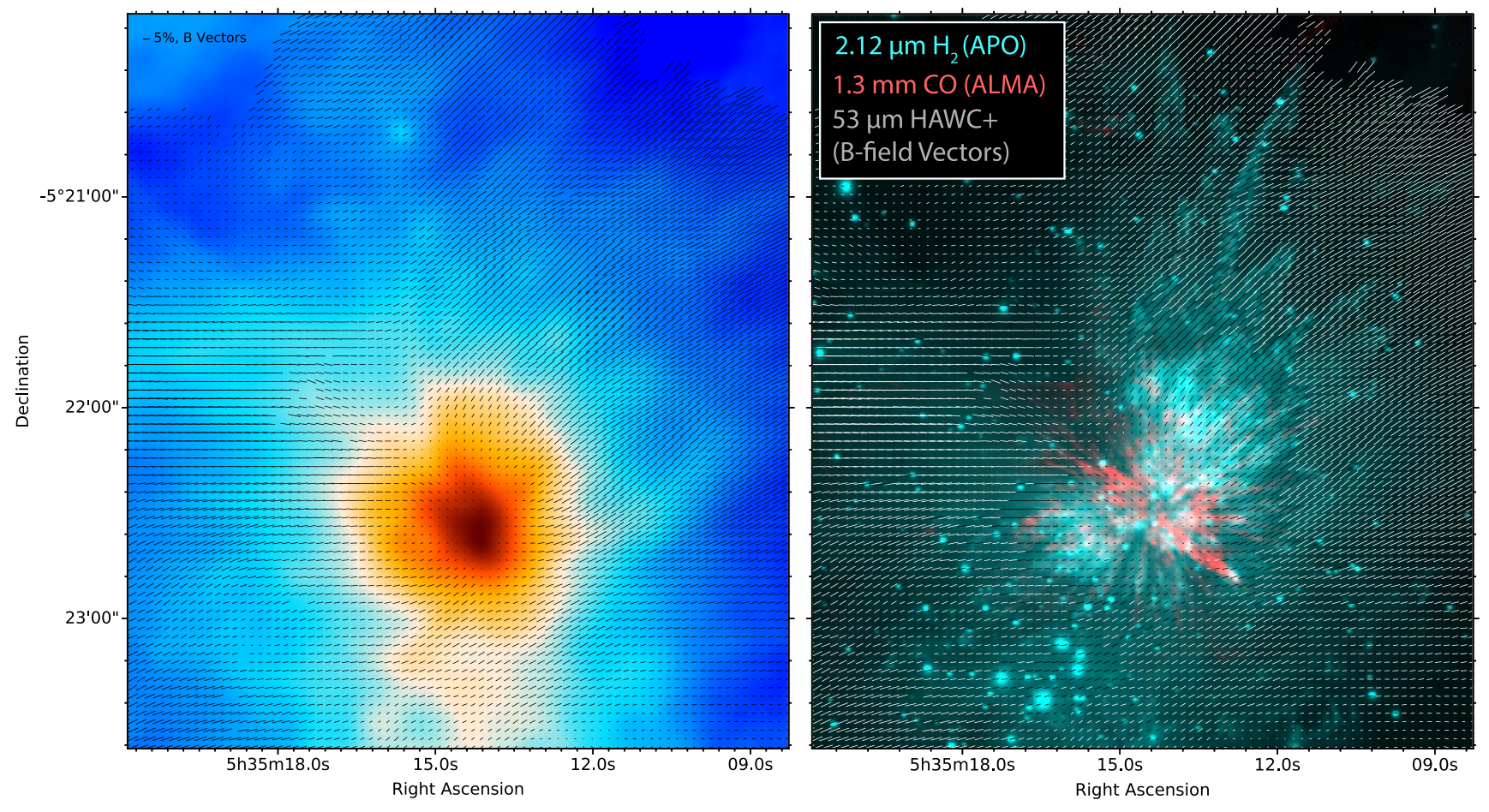

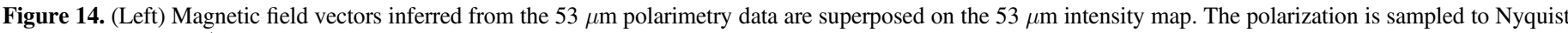

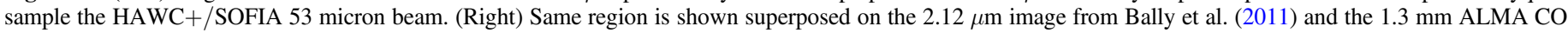
map (Bally et al. 2017). The geometry of the magnetic field inferred by the band A polarimetry traces the bipolar outflow.

structure of the field and the $\mathrm{H}_{2}$ gas in the outer regions of the explosion.

This argument assumes that the characteristic field inferred from the DCF technique is approximately uniform over the $\mathrm{BNKL}$ region. Zeeman measurements of $\mathrm{H}_{2} \mathrm{O}$ masers have indicated fields up to $\sim 40 \mathrm{mG}$ near the infrared source IRc2 near the center of the BNKL region (Genzel \& Downes 1977; Fiebig \& Guesten 1989; Garay et al. 1989), indicating that the details of the magnetic interaction are more complicated than the simple picture presented here. However, these large fields are likely confined to very small volumes of high-density material and unlikely to significantly affect the dynamics in the volumes discussed here. Future polarimetric observations with ALMA may be able to shed more light on the details.

\section{Summary}

We have obtained new continuum far-infrared polarimetric and photometric maps of the OMC-1 region at 53, 89, 154, and $214 \mu \mathrm{m}$ using the HAWC + instrument on SOFIA.

1. We have produced new maps of the temperatures and column densities of this region by combining HAWC+ photometry in four bands with other data sets.

2. The magnetic field geometry inferred from the polarization at these wavelengths indicates a similar large-scale field oriented roughly perpendicular to the $\mathrm{BN} / \mathrm{KL}$ cloud as seen in previous studies. However, at the shorter (53, $89 \mu \mathrm{m})$ wavelengths, the magnetic field structure around the $\mathrm{BN}$ object shows a similar bipolar structure to molecular tracers of the $\mathrm{BN} / \mathrm{KL}$ explosion.

3. Analysis of the polarization fraction as a function of the local dispersion of the polarization vectors provides no evidence for loss of grain alignment within the cloud.
This could be due to stronger radiation fields in the OMC1 region than in other Milky Way star formation regions.

4. We estimate the magnetic field strength in the bar and $\mathrm{H}$ II regions of the OMC-1 to be $\sim 250-300 \mu \mathrm{G}$.

5. Statistical estimates for the magnetic field indicate values of the field strength in th $\mathrm{BN} / \mathrm{KL}$ region to be $\sim 1 \mathrm{mG}$, consistent with a picture in which the explosion dominates the magnetic field near the center, but the kinetic and magnetic energy densities are close to equipartition in the outer regions of the explosion.

Based on observations made with the NASA/DLR Stratospheric Observatory for Infrared Astronomy (SOFIA). SOFIA is jointly operated by the Universities Space Research Association, Inc. (USRA), under NASA contract NAS297001, and the Deutsches SOFIA Institut (DSI) under DLR contract 50 OK 0901 to the University of Stuttgart. Financial support for this work was provided by NASA through awards \#SOF 05-0038 and \#SOF 05-0018 issued by USRA.

The authors would like to thank Joe Adams for skillful operation of $\mathrm{HAWC}+$, SOFIA observatory personnel for the successful research flights leading to the OMC-1 results, Melanie Clarke for collaborative work on the HAWC + data analysis pipeline, and Rahul Thapa and Lexi Tumblety for assistance with figure preparation and analysis. The authors would like to thank Simon Coudé, Steve Mairs, the JCMT Gould Belt Legacy Survey, and the East Asian Observatory (EAO) for their assistance in locating and using the CO-subtracted GBS data. The authors would like to thank Simon Dicker and Brian Mason for supplying the MUSTANG and $X$-band data.

Parts of the analysis were performed using the Clusty Computing Facility in the Villanova Department of 
Astrophysics and Planetary Science. We thank Andrej Prša for his support in leading and maintaining this resource.

PACS has been developed by a consortium of institutes led by MPE (Germany) and including UVIE (Austria); KU Leuven, CSL, IMEC (Belgium); CEA, LAM (France); MPIA (Germany); INAFIFSI/OAA/OAP/OAT, LENS, SISSA (Italy); and IAC (Spain). This development has been supported by the funding agencies BMVIT (Austria), ESA-PRODEX (Belgium), CEA/CNES (France), DLR (Germany), ASI/INAF (Italy), and CICYT/MCYT (Spain). SPIRE has been developed by a consortium of institutes led by Cardiff University (UK) and including the University of Lethbridge (Canada); NAOC (China); CEA, LAM (France); IFSI, the University of Padua (Italy); IAC (Spain); Stockholm Observatory (Sweden); Imperial College London, RAL, UCLMSSL, UKATC, Univ. Sussex (UK); and Caltech, JPL, NHSC, the University of Colorado (USA). This development has been supported by national funding agencies: CSA (Canada); NAOC (China); CEA, CNES, CNRS (France); ASI (Italy); MCINN (Spain); SNSB (Sweden); STFC, UKSA (UK); and NASA (USA). HCSS/HSpot/HIPE is a joint development by the Herschel Science Ground Segment Consortium, consisting of ESA, the NASA Herschel Science Center, and the HIFI, PACS, and SPIRE consortia. This work is based in part on observations made with Herschel, a European Space Agency Cornerstone Mission with significant participation by NASA. Portions of this work were carried out at the Jet Propulsion Laboratory, operated by the California Institute of Technology under a contract with NASA. The James Clerk Maxwell Telescope has historically been operated by the Joint Astronomy Centre on behalf of the Science and Technology Facilities Council of the United Kingdom, the National Research Council of Canada and the Netherlands Organisation for Scientific Research. Additional funds for the construction of SCUBA-2 were provided by the Canada Foundation for Innovation. This paper made use of SCUBA-2 data taken as part of program ID MJLSG31.

We would like to thank the anonymous referee for helpful comments.

Software: python, Ipython (Pérez \& Granger 2007), numpy (van der Walt et al. 2011), scipy (Jones et al. 2001) matplotlib (Hunter 2007), emcee (Foreman-Mackey et al. 2013), corner (Foreman-Mackey 2016), astropy (Astropy Collaboration et al. 2013; Price-Whelan et al. 2018), LIC code (ported from public available IDL source by Diego FalcetaGonçalves).

\section{ORCID iDs}

David T. Chuss @i https://orcid.org/0000-0003-0016-0533 B-G Andersson (1) https://orcid.org/0000-0001-6717-0686 John Bally (1) https://orcid.org/0000-0001-8135-6612 Jessie L. Dotson (i) https://orcid.org/0000-0003-4206-5649 Jordan A. Guerra (i) https://orcid.org/0000-0001-8819-9648 Martin Houde (1) https://orcid.org/0000-0003-4420-8674 Terry Jay Jones (i) https://orcid.org/0000-0002-8716-6980 Enrique Lopez Rodriguez 1 i https://orcid.org/0000-00015357-6538

Joseph M. Michail (1) https://orcid.org/0000-0003-3503-3446 Mark R. Morris (i) https://orcid.org/0000-0002-6753-2066 Giles Novak (1) https://orcid.org/0000-0003-1288-2656 Johannes Staguhn (1) https://orcid.org/0000-0002-8437-0433 John E. Vaillancourt (i) https://orcid.org/0000-00018916-1828
Edward J. Wollack (i) https://orcid.org/0000-0002-7567-4451

Dominic J. Benford (ㄷ) https://orcid.org/0000-0002-9884-4206

Erin G. Cox (1) https://orcid.org/0000-0002-5216-8062

Daniel A. Dale (1) https://orcid.org/0000-0002-5782-9093

L. M. Fissel (1) https://orcid.org/0000-0002-4666-609X

Paul F. Goldsmith (i) https://orcid.org/0000-0002-6622-8396

Ryan T. Hamilton (1) https://orcid.org/0000-0001-6350-2209

Shaul Hanany (1) https://orcid.org/0000-0002-8702-6291

Leslie W. Looney (1) https://orcid.org/0000-0002-4540-6587

Fabio P. Santos (ib https://orcid.org/0000-0002-9650-3619

Ian Stephens iㅏ https://orcid.org/0000-0003-3017-4418

Konstantinos Tassis (1) https://orcid.org/0000-0002-8831-2038 Derek Ward-Thompson (i) https://orcid.org/0000-0003-

1140-2761

\section{References}

Abergel, A. 2010, Herschel Space Observatory Proposal 285, SDP_aabergel_3: Evolution of Interstellar Dust (Paris: ESA)

Allen, D. A., \& Burton, M. G. 1993, Natur, 363, 54

Andersson, B. G., Lazarian, A., \& Vaillancourt, J. E. 2015, ARA\&A, 53, 501

André, P. 2007, KPGT_pandre_1: Probing the Origin of the Stellar Initial Mass Function: A Wide-field Herschel Photometric Survey of Nearby Starforming Cloud Complexes, Herschel Space Observatory Proposal

André, P. 2011, GT2_pandre_5: Completion of the Gould Belt and HOBYS Surveys, Herschel Space Observatory Proposal

Arab, H., Abergel, A., Habart, E., et al. 2012, A\&A, 541, A19

Astropy Collaboration, Robitaille, T. P., Tollerud, E. J., et al. 2013, A\&A, 558, A33

Bally, J., Cunningham, N. J., Moeckel, N., et al. 2011, ApJ, 727, 113

Bally, J., Ginsburg, A., Arce, H., et al. 2017, ApJ, 837, 60

Bally, J., \& Zinnecker, H. 2005, AJ, 129, 2281

Becklin, E. E., \& Neugebauer, G. 1967, ApJ, 147, 799

Bendo, G. J., Griffin, M. J., Bock, J. J., et al. 2013, MNRAS, 433, 3062

Benoît, A., Ade, P., Amblard, A., et al. 2004, A\&A, 424, 571

Bernard, J. P., Paradis, D., Marshall, D. J., et al. 2010, A\&A, 518, L88

Buckle, J. V., Hills, R. E., Smith, H., et al. 2009, MNRAS, 399, 1026

Cabral, B., \& Leedom, L. C. 1993, in Proc. 20th Annual Conf. Comp. Graph. Interact. Tech. (New York: ACM), 263, https://dl.acm.org/citation.cfm? $\mathrm{id}=166151$

Chandrasekhar, S., \& Fermi, E. 1953, ApJ, 118, 113

Chapin, E. L., Berry, D. S., Gibb, A. G., et al. 2013, MNRAS, 430, 2545

Coudé, S., Bastien, P., Kirk, H., et al. 2016, MNRAS, 457, 2139

Davis, L. 1951, PhRv, 81, 890

Dempsey, J. T., Friberg, P., Jenness, T., et al. 2013, MNRAS, 430, 2534

Dicker, S. R., Korngut, P. M., Mason, B. S., et al. 2008, Proc. SPIE, 7020, 702005

Dicker, S. R., Mason, B. S., Korngut, P. M., et al. 2009, ApJ, 705, 226

Dolginov, A. Z., \& Mytrophanov, I. G. 1976, Ap\&SS, 43, 291

Dotson, J. L. 1996, ApJ, 470, 566

Dowell, C. D. 1997, ApJ, 487, 237

Draine, B. T., \& Hensley, B. S. 2017, ApJ, submitted (arXiv:1710.08968)

Draine, B. T., \& Weingartner, J. C. 1997, ApJ, 480, 633

Dupac, X., Bernard, J. P., Boudet, N., et al. 2003, A\&A, 404, L11

Dupac, X., Giard, M., Bernard, J. P., et al. 2001, ApJ, 553, 604

Fiebig, D., \& Guesten, R. 1989, A\&A, 214, 333

Fissel, L. M., Ade, P. A. R., Angilè, F. E., et al. 2016, ApJ, 824, 134

Foreman-Mackey, D., Hogg, D. W., Lang, D., \& Goodman, J. 2013, PASP, 125,306

Foreman-Mackey, D. 2016, JOSS, 1, 24

Galametz, M., Kennicutt, R. C., Albrecht, M., et al. 2012, MNRAS, 425, 763

Galametz, M., Maury, A., Girart, J. M., et al. 2018, A\&A, 618, A139

Gandilo, N. N., Ade, P. A. R., Angilè, F. E., et al. 2016, ApJ, 824, 84

Garay, G., Moran, J. M., \& Haschick, A. D. 1989, ApJ, 338, 244

Genzel, R., \& Downes, D. 1977, A\&A, 61, 117

Griffin, M. J., Abergel, A., Abreu, A., et al. 2010, A\&A, 518, L3

Guillet, V., Fanciullo, L., Verstraete, L., et al. 2018, A\&A, 610, A16

Harper, D. A., Runyan, M. C., Dowell, C. D., et al. 2018, JAI, 7, 1840008

Heiles, C., Goodman, A. A., McKee, C. F., \& Zweibel, E. G. 1993, in Protostars and Planets III, ed. E. H. Levy \& J. I. Lunine (Tucson, AZ: Univ. Arizona Press), 279

Hensley, B., Murphy, E., \& Staguhn, J. 2015, MNRAS, 449, 809 
Hildebrand, R. H., Davidson, J. A., Dotson, J. L., et al. 2000, PASP, 112, 1215

Hildebrand, R. H., Dotson, J. L., Dowell, C. D., Schleuning, D. A., \& Vaillancourt, J. E. 1999, ApJ, 516, 834

Hildebrand, R. H., \& Dragovan, M. 1995, ApJ, 450, 663

Hildebrand, R. H., Kirby, L., Dotson, J. L., Houde, M., \& Vaillancourt, J. E. 2009, ApJ, 696, 567

Hill, T., Motte, F., Didelon, P., et al. 2011, A\&A, 533, A94

Holland, W. S., Bintley, D., Chapin, E. L., et al. 2013, MNRAS, 430, 2513

Houde, M., Dowell, C. D., Hildebrand, R. H., et al. 2004, ApJ, 604, 717

Houde, M., Hull, C. L. H., Plambeck, R. L., Vaillancourt, J. E., \& Hildebrand, R. H. 2016, ApJ, 820, 38

Houde, M., Rao, R., Vaillancourt, J. E., \& Hildebrand, R. H. 2011, ApJ, 733, 109

Houde, M., Vaillancourt, J. E., Hildebrand, R. H., Chitsazzadeh, S., \& Kirby, L. 2009, ApJ, 706, 1504

Hunter, J. D. 2007, CSE, 9, 90

Johnston, K. J., Migenes, V., \& Norris, R. P. 1989, ApJ, 341, 847

Jones, E., Oliphant, T., Peterson, P., et al. 2001, SciPy: Open Source Scientific Tools for Python, http://www.scipy.org/

Jones, T. J. 1989, ApJ, 346, 728

Jones, T. J., Bagley, M., Krejny, M., Andersson, B. G., \& Bastien, P. 2015, AJ, 149,31

Jones, T. J., \& Whittet, D. C. B. 2015, in Interstellar Polarization, ed L. Kolokolova, J. Hough, \& A. Levasseur-Regourd (Cambridge: Cambridge Univ. Press), 147

Kleinmann, D. E., \& Low, F. J. 1967, ApJL, 149, L1

Kobulnicky, H. A., Molnar, L. A., \& Jones, T. J. 1994, AJ, 107, 1433

Kounkel, M., Hartmann, L., Loinard, L., et al. 2017, ApJ, 834, 142

Kovács, A. 2006, PhD thesis, Caltech Univ.

Kovács, A. 2008, Proc. SPIE, 7020, 70201

Lazarian, A., \& Hoang, T. 2007, MNRAS, 378, 910

Mairs, S., Johnstone, D., Kirk, H., et al. 2016, MNRAS, 461, 4022

Müller, T., Okumura, K., \& Klaas, U. 2011, PACS Photometer Passbands and Colour Correction Factors for Various Source SEDs (Paris: ESA), https:// www.cosmos.esa.int/documents/12133/996891/PACS+Photometer + Passbands + and + Colour + Correction + Factors + for + Various + Source + SEDs

Myers, P. C., \& Goodman, A. A. 1991, ApJ, 373, 509
Novak, G. 2011, in ASP Conf. Ser. 449, Astronomical Polarimetry 2008: Science from Small to Large Telescopes, ed. P. Bastien et al. (San Francisco, CA: ASP), 50

Novak, G., Dotson, J. L., Dowell, C. D., et al. 1997, ApJ, 487, 320

Novak, G., Dotson, J. L., Dowell, C. D., et al. 2000, ApJ, 529, 241

Ott, S. 2010, in ASP Conf. Ser. 434, Astronomical Data Analysis Software and Systems XIX, ed. Y. Mizumoto, K.-I. Morita, \& M. Ohishi (San Francisco, CA: ASP), 139

Pattle, K., Ward-Thompson, D., Berry, D., et al. 2017, ApJ, 846, 122

Pérez, F., \& Granger, B. E. 2007, CSE, 9, 21

Pilbratt, G. L., Riedinger, J. R., Passvogel, T., et al. 2010, A\&A, 518, L1

Planck Collaboration, Ade, P. A. R., Aghanim, N., et al. 2015, A\&A, 576, A104

Planck Collaboration, Aghanim, N., Akrami, Y., et al. 2018, arXiv:1807.06212

Poglitsch, A., Waelkens, C., Geis, N., et al. 2010, A\&A, 518, L2

Poidevin, F., Bastien, P., \& Jones, T. J. 2011, ApJ, 741, 112

Price-Whelan, A. M., Sipócz, B. M., Günther, H. M., et al. 2018, AJ, 156, 123

Roussel, H. 2013, PASP, 125, 1126

Sadavoy, S. I., Di Francesco, J., Johnstone, D., et al. 2013, ApJ, 767, 126

Schleuning, D. A. 1998, ApJ, 493, 811

Serkowski, K. 1974, in Methods in Experimental Physics, ed. N. Carleton (New York: Academic), 361

Shetty, R., Kauffmann, J., Schnee, S., Goodman, A. A., \& Ercolano, B. 2009, ApJ, 696, 2234

Snell, R. L., Scoville, N. Z., Sanders, D. B., \& Erickson, N. R. 1984, ApJ, 284, 176

Soler, J. D., Hennebelle, P., Martin, P. G., et al. 2013, ApJ, 774, 128

Tang, Y.-W., Ho, P. T. P., Koch, P. M., \& Rao, R. 2010, ApJ, 717, 1262

Vaillancourt, J. E. 2002, ApJS, 142, 53

Vallée, J. P., \& Bastien, P. 1999, ApJ, 526, 819

Valtchanov, I. 2017, Herschel Explanatory Supplement volume IV: The Spectral and Photometric Imaging Receiver (SPIRE) Handbook (Paris: ESA), http://herschel.esac.esa.int/Docs/SPIRE/html/spire_om.html van der Walt, S., Colbert, S. C., \& Varoquaux, G. 2011, CSE, 13, 22

Ward-Thompson, D., Pattle, K., Bastien, P., et al. 2017, ApJ, 842, 66 